\title{
THE LAS CAMPANAS INFRARED SURVEY. III. THE $H$-BAND IMAGING SURVEY AND THE NEAR-INFRARED AND OPTICAL PHOTOMETRIC CATALOGS
}

\author{
Hsiao-Wen Chen, ${ }^{1}$ P. J. McCarthy, ${ }^{1}$ R. O. Marzke ${ }^{1,2}$ J. Wilson, ${ }^{1}$ R. G. Carlberg, ${ }^{3}$ A. E. Firth, ${ }^{4}$ S. E. Persson, ${ }^{1}$ \\ C. N. Sabbey, ${ }^{4}$ J. R. Lewis, ${ }^{4}$ R. G. McMahon, ${ }^{4}$ O. Lahav, ${ }^{4}$ R. S. Ellis, ${ }^{5}$ P. Martini, ${ }^{1}$ R. G. Abraham, ${ }^{3}$ A. Oemler, ${ }^{1}$ \\ D. C. Murphy, ${ }^{1}$ R. S. Somerville ${ }^{4}$ M. G. Beckett, ${ }^{1,4}$ and C. D. Mackay ${ }^{4}$ \\ Received 2001 August 2; accepted 2002 January 2
}

\begin{abstract}
The Las Campanas Infrared Survey, based on broadband optical and near-infrared photometry, is designed to robustly identify a statistically significant and representative sample of evolved galaxies at redshifts $z>1$. We have completed an $H$-band imaging survey over $1.1 \mathrm{deg}^{2}$ of sky in six separate fields. The average $5 \sigma$ detection limit in a $4^{\prime \prime}$ diameter aperture is $H \sim 20.8$. Here we describe the design of the survey, the observation strategies, data-reduction techniques, and object identification procedures. We present sample near-infrared and optical photometric catalogs for objects identified in two survey fields. The optical images of the Hubble Deep Field-South region obtained from the literature reach $5 \sigma$ detection thresholds in a 4" diameter aperture of $U \sim 24.6, B \sim 26.1, V \sim 25.6, R \sim 25.1$, and $I \sim 24.2 \mathrm{mag}$. The optical images of the Chandra Deep Field-South region obtained from our own observations reach $5 \sigma$ detection thresholds in a $4^{\prime \prime}$ diameter aperture of $V \sim 26.8, R \sim 26.2, I \sim 25.3$, and $z^{\prime} \sim 23.7$ mag. We perform object detection in all bandpasses and identify $\gtrsim 54,000$ galaxies over $1408 \mathrm{arcmin}^{2}$ of sky in the two fields. Of these galaxies, $\sim 14,000$ are detected in the $H$ band and $\sim 2000$ have the colors of evolved galaxies, $I-H \gtrsim 3$, at $z \gtrsim 1$. We find that (1) the differential number counts $N(m)$ for the $H$-band-detected objects has a slope of $d \log N(m) / d m=0.45 \pm 0.01 \mathrm{mag}^{-2}$ at $H \lesssim 19$ and $0.27 \pm 0.01 \mathrm{mag}^{-2}$ at $H \gtrsim 19$, with a mean surface density $\approx 7200 \mathrm{deg}^{-2} \mathrm{mag}^{-1}$ at $H=19$. In addition, we find that (2) the differential number counts for the $H$-banddetected red objects has a very steep slope, $d \log N(m ; I-H \gtrsim 3) / d m=0.84 \pm 0.06 \mathrm{mag}^{-2}$ at $H \lesssim 20$ and $0.32 \pm 0.07 \mathrm{mag}^{-2}$ at $H \gtrsim 20$, with a mean surface density $\approx 3000 \mathrm{deg}^{-2} \mathrm{mag}^{-1}$ at $H=20$. Finally, we find that (3) galaxies with red optical to near-IR colors $(I-H>3)$ constitute $\approx 20 \%$ of the $H$-band-detected galaxies at $H \lesssim 21$, but only $\approx 2 \%$ at $H \lesssim 19$. We show that red galaxies are strongly clustered, which results in a strong field-to-field variation in their surface density. Comparisons of observations and predictions based on various formation scenarios indicate that these red galaxies are consistent with mildly evolving early-type galaxies at $z \sim 1$, although with a significant amount of ongoing star formation, as indicated by the large scatter in their $V-I$ colors.
\end{abstract}

Subject headings: catalogs — cosmology: observations — galaxies: evolution — galaxies: high-redshift — infrared: galaxies - surveys

On-line material: machine-readable tables

\section{INTRODUCTION}

Different galaxy formation scenarios provide distinct predictions for the space densities and masses of evolved galaxies at redshifts beyond $z=1$. In monolithic collapse scenarios, massive galaxies form early over a dynamical time (e.g., Eggen, Lynden-Bell, \& Sandage 1962) and passively evolve to the present epoch. The comoving space density of evolved galaxies is therefore expected to remain constant, and the intrinsic luminosities of these galaxies are expected to increase gradually with increasing redshift. In hierarchical formation scenarios, massive galaxies form later through mergers of low-mass galaxies over a significant fraction of the Hubble time (White \& Rees 1978). The

\footnotetext{
${ }^{1}$ Carnegie Observatories, 813 Santa Barbara Street, Pasadena, CA 91101.

${ }^{2}$ Department of Astronomy, University of Toronto, Toronto, ON M5S 3H8, Canada.

${ }^{3}$ Department of Astronomy and Physics, San Francisco State University, San Francisco, CA 94132-4163.

${ }^{4}$ Institute of Astronomy, Cambridge CB3 OHA, England, UK.

${ }^{5}$ Department of Astronomy, California Institute of Technology 105-24, Pasadena, CA 91125.
}

bulk of star formation and mass assembly occurs much later, and the comoving space density of evolved galaxies is expected to decline steeply with redshift at $z>1$. While various surveys have identified some luminous galaxy populations at $z>2$ (e.g., Steidel et al. 1999; Blain et al. 1999), the connection between these galaxies and typical present-day galaxies is unclear. On the other hand, comparisons of statistical properties of evolved high-redshift galaxies and local elliptical galaxies may provide a direct means of discriminating between competing galaxy formation scenarios (e.g., Kauffmann \& Charlot 1998).

Evolved galaxies can be characterized by their intrinsically red colors due to the lack of ongoing star formation, which provides most of the UV light in typical galaxy spectral energy distributions. At redshifts beyond unity, where the UV region of the spectrum is redshifted to the visible, optical galaxy surveys are insensitive to evolved galaxies, because of their steep UV spectra. Identifications of evolved high-redshift galaxies therefore rely on observations carried out at near-infrared wavelengths. Various studies based on near-infrared surveys have, however, yielded inconsistent measurements of the space density of evolved galaxies (Kauffmann, Charlot, \& White 1996; Totani \& Yoshii 1998; 
Zepf 1997; Franceschini et al. 1998; Benítez et al. 1999; Menanteau et al. 1999; Barger et al. 1999; Schade et al. 1999; Broadhurst \& Bouwens 2000; Daddi et al. 2000; Martini 2001a). Because most of the existing deep, near-infrared surveys observe a sky area of only a few tens of square arcminutes, the discrepancy is likely due to significant field-tofield variation (e.g., Daddi et al. 2000; McCarthy et al. 2001a, 2001b). Furthermore, because evolved galaxies lack prominent narrowband spectral features in the UV spectral range, this discrepancy is also likely due to selection biases (e.g., Totani \& Yoshii 1998). Additional uncertainty may also arise as a result of the presence of dusty star-forming galaxies that exhibit red colors resembling evolved high-redshift galaxies (e.g., Graham \& Dey 1996; Smail, Ivison, \& Blain 1997).

A complete survey of evolved galaxies over a large sky area is needed to address these issues. Deep wide-field nearinfrared surveys have only recently become feasible with the advent of large-format near-infrared cameras (Beckett et al. 1998; Elston 1998; Persson et al. 2001). In addition, various groups have demonstrated that distant galaxies can be accurately and reliably identified using photometric redshift techniques that incorporate optical and near-infrared broadband photometry (Connolly et al. 1997; Sawicki, Lin, \& Yee 1997; Lanzetta, Fernández-Soto, \& Yahil 1998; Fernández-Soto, Lanzetta, \& Yahil 1999; Fernández-Soto et al. 2001). For galaxies lacking strong emission or absorption features, we may still be able to estimate redshifts based on the presence/absence of spectral discontinuities using photometric redshift techniques. Therefore, a wide-field optical and near-infrared imaging survey, with sufficiently accurate colors to yield reliable photometric redshifts, offers an opportunity to identify statistically significant and representative samples of evolved galaxies at $z>1$.

We initiated the Las Campanas Infrared (LCIR) Survey to obtain and analyze deep near-infrared images and complementary optical images in the $V, R, I$, and $z^{\prime}$ bandpasses over $1 \mathrm{deg}^{2}$ of sky at high Galactic latitudes (Marzke et al. 1999; McCarthy et al. 2001a, 2001b). The survey is designed to identify a large number of red galaxies at $z>1$, while securing a uniform sample of galaxies of all types to $z \sim 2$ using broadband optical and near-infrared colors. In particular, this program utilizes one of the largest near-infrared cameras available (CIRSI), which produces an image of $13^{\prime} \times 13^{\prime}$ contiguous field of view in a sequence of four pointings. The LCIR survey may serve to bridge the gap between very wide field surveys (e.g., NOAO Deep-Wide) ${ }^{6}$ and very deep, smaller field near-infrared imaging surveys (e.g., ESO Imaging Survey, da Costa 20001; NTT Deep Field, Moorwood, Cuby, \& Lidman 1998).

The primary objectives of the program are (1) to examine the nature of the red galaxy population and identify evolved galaxies at redshifts $z>1$, (2) to study the space density and luminosity evolution of evolved galaxies at redshifts $z \lesssim 2$, and (3) to measure the spatial clustering of evolved highredshift galaxies (McCarthy et al. 2001a, 2001b; Firth et al. 2002 , in preparation), thereby inferring merging rates of these galaxies for constraining theoretical models. The galaxy catalog together with an accompanied photometric redshift catalog will also be used (4) to assess luminosity and luminosity density evolution for galaxies of different types over the redshift range between $z=0$ and 2 (H.-W. Chen et al., in preparation) and (5) to study the bright-end luminosity functions for galaxies and QSOs at redshifts beyond $z=4.5$. Finally, galaxies identified in the survey will be compared with objects identified in a companion VLA survey (6) to study the spatial distribution of weak radio sources ( $\gtrsim 10 \mu \mathrm{Jy} ; \mathrm{L}$. Yan et al., in preparation).

To date, we have completed an $H$-band imaging survey over $1.1 \mathrm{deg}^{2}$ of sky to a mean $5 \sigma$ detection limit in a $4^{\prime \prime}$ diameter aperture of $H \sim 20.8$. Here we present sample optical and near-infrared photometric catalogs for galaxies identified in two of our fields: the Hubble Deep Field-South (HDF-S) and Chandra Deep Field-South (CDF-S). These are the two fields for which a complete set of near-infrared and optical images are available. We have identified $\sim 24,000$ galaxies over $847 \mathrm{arcmin}^{2}$ in the HDF-S region, of which $\sim 6720$ are detected in the $H$-band survey, and $\sim 30,000$ galaxies over $561 \operatorname{arcmin}^{2}$ in the CDF-S region, of which $\sim 7400$ are detected in the $H$-band survey. Among the $H$-detected galaxies in the two regions, $\sim 2000$ have colors that match evolved galaxies, $I-H \gtrsim 3$, at redshifts $z \gtrsim 1$. A complete near-infrared and optical photometric catalog of the two fields is available electronically. ${ }^{7}$ Catalogs for the other survey fields will be released as they become available.

We describe the survey design and observation strategies in $\S 2$ and data reduction and image processing in $\S 3$. The procedures for identifying objects in multi-bandpasses, estimates of the completeness limits of the catalogs, and an assessment of the reliability of the photometric measurements are presented in $\S 4$. The format of the optical and near-infrared photometric catalogs is described in $\S 5$. Finally, we discuss statistical properties of the $H$-bandselected galaxies in $\S 6$. An independent analysis based on objects identified in the HDF-S region is also presented in Firth et al. (2002, in preparation). We adopt the cosmology $\Omega_{M}=0.3$ and $\Omega_{\Lambda}=0.7$, with a dimensionless Hubble constant $h=H_{0} /\left(100 \mathrm{~km} \mathrm{~s}^{-1} \mathrm{Mpc}^{-1}\right)$ throughout the paper.

\section{OBSERVATIONS}

\subsection{Definition of the Las Campanas Infrared Survey}

The goals of the LCIR survey are to robustly identify a statistically significant and representative sample of evolved galaxies at redshifts $z>1$, as well as to secure a uniform sample of galaxies of all types at intermediate redshifts based on broadband optical and near-infrared colors.

To determine the image depths necessary to reach our goals, we estimated the expected near-infrared brightness and optical and near-infrared colors of evolved high-redshift galaxies using a series of evolutionary models. We considered a nonevolving model using an empirical early-type galaxy template presented in Coleman, $\mathrm{Wu}, \&$ Weedman (1980) and two evolving models for galaxies of solar metallicity formed in a single burst of 1 Gyr duration at redshifts $z_{f}=5$ and 10, respectively (Bruzual \& Charlot 1993). We scaled these models to have $L_{H}=L_{H_{*}}$ at $z=0$ (see also Marzke et al. 1999). ${ }^{8}$ Figure 1 shows the predicted redshift evolution of the observed $H$-band magnitudes and optical

\footnotetext{
${ }^{6}$ NOAO Deep-Wide is available at http:// www.noao.edu/noao/noaodeep.
} 

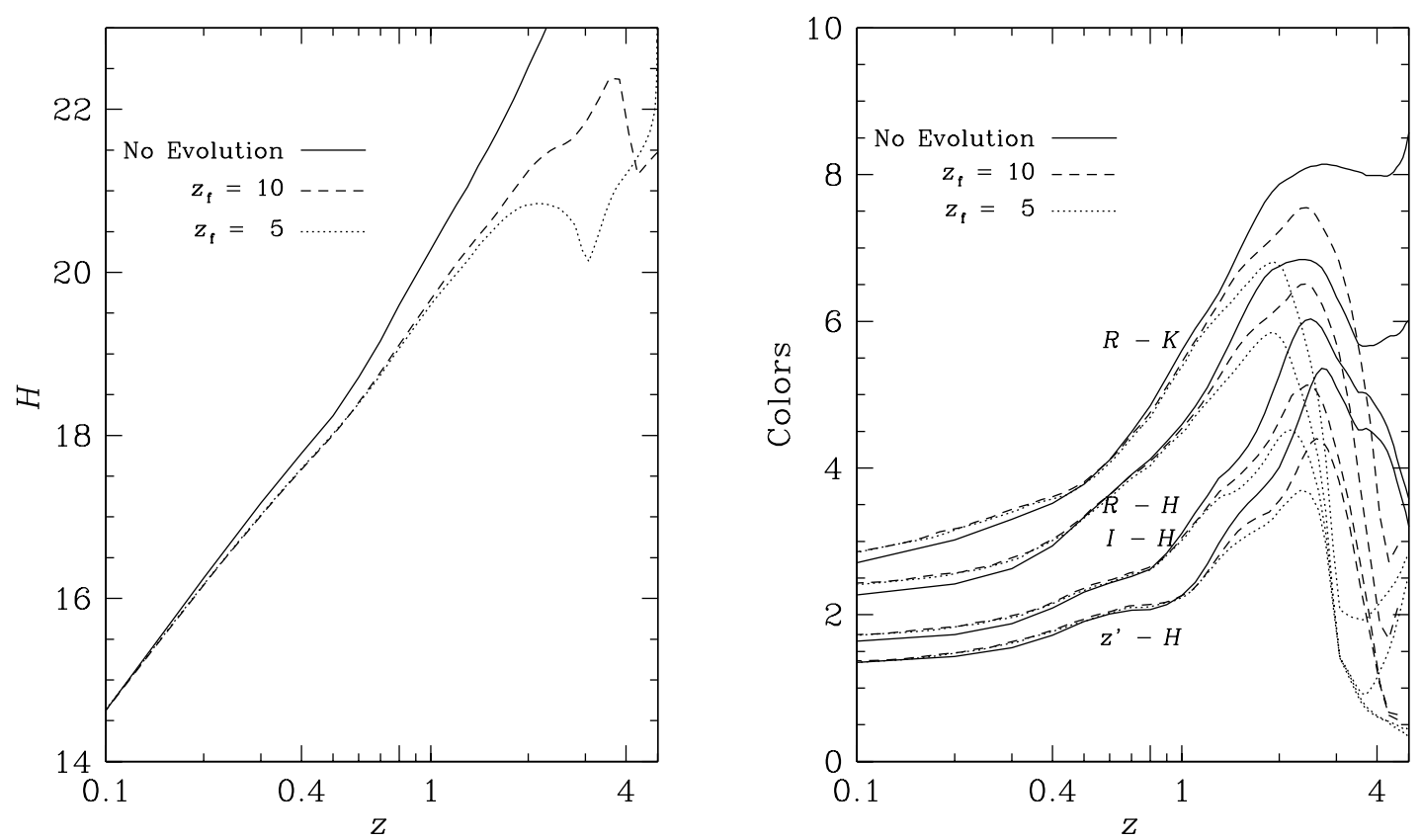

Fig. 1.-Predicted $H$-band magnitude (left $)$ and optical and near-infrared colors (right) of an $L_{*}$ elliptical galaxy as a function of redshift. The solid curves are for the nonevolving model; the dashed curves are for a single burst galaxy formed at $z_{f}=10$; the dotted curves are for a single burst galaxy formed at $z_{f}=5$. The curves of the right panel represent the redshift evolution of (top to bottom) the $R-K, R-H, I-H$, and $z^{\prime}-H$ colors. It is shown that evolved galaxies at redshifts $z>1$ can be selected based on $R-K \gtrsim 6, R-H \gtrsim 4.5, I-H \gtrsim 3$, or $z^{\prime}-H \gtrsim 2$.

and near-infrared colors of evolved galaxies based on the three models. Adopting $H_{0}=70 \mathrm{~km} \mathrm{~s}^{-1} \mathrm{Mpc}^{-1}$, we found that in the absence of dust an evolved $L_{*}$ galaxy can have an apparent $H$-band magnitude between $H=19.5$ and 20.3 and $I-H \sim 3$ at redshift $z \sim 1$, and an apparent $H$-band magnitude between $H=20.7$ and 22.5 and an $I-H$ color between $\sim 4.5$ and 5 at redshift $z \sim 2$. The color threshold $I-H \gtrsim 3$ therefore defines our selection criterion for evolved galaxies at redshifts $z>1$, which according to Figure 1 is similar to selections made based on $R-K_{s} \gtrsim 5$. To identify a large number of the evolved galaxy population, we therefore choose a target $5 \sigma$ survey depth of $H \sim 21$, more than 1 mag fainter than an $L_{*}$ elliptical galaxy at $z \sim 1$. We also require that the optical imaging survey reach consistent depths based on the predicted optical and near-infrared colors. The actual depth that we achieved varied from field to field and often fell somewhat short of our design goals.

To determine the survey area, we first estimated the number of evolved galaxies that are required to obtain a significant signal in the clustering analysis. At a projected comoving correlation length $r_{p}=1 \mathrm{Mpc}$, we found that a sample of $\sim 3000$ galaxies are needed to reach the $5 \sigma$ level of significance (Marzke et al. 1999). The surface density of galaxies satisfying $I-H \gtrsim 3$ is, however, very uncertain. Different measurements based on $K$-band surveys have been reported by several groups, ranging from 0.3 to $3 \mathrm{arcmin}^{-2}$ at $K_{s}<20$ (Cowie et al. 1994; Barger et al. 1999). Adopting a median surface density 1 arcmin $^{-2}$ and a mean near-infrared color $H-K_{s} \sim 1$ for evolved galaxies at $z>1$, we concluded that $1 \mathrm{deg}^{2}$ of sky at a $5 \sigma$ limiting magnitude $H \sim 21$ will yield $\sim 3000$ red high-redshift galaxies.

The LCIR survey may serve to bridge the gap between the ongoing NOAO Wide-Field Survey that images $18 \mathrm{deg}^{2}$ of sky at a shallower depth (see NOAO Deep-Wide Web page) and the existing deep, smaller field near-infrared imaging surveys such as the NTT Deep Field (Moorwood et al. 1998). We have selected six distinct equatorial and southern fields at high Galactic latitudes distributed in right ascension. In Table 1, we list the fields, their J2000 coordinates, and Galactic latitudes.

\subsection{Infrared Imaging Observations}

The $H$-band imaging survey was carried out at the du Pont $2.5 \mathrm{~m}$ telescope at Las Campanas using the Cambridge Infrared Survey Instrument (CIRSI). This instrument contains a sparse mosaic of four $1024 \times 1024$ Rockwell Hawaii HgCdTe arrays (Beckett et al. 1998; Persson et al. 2001). At the Cassegrain focus of the du Pont telescope, the plate scale of the camera is 0.199 pixel $^{-1}$. Some of the observations were made with a doublet field corrector in front of the dewar window, which produced a plate scale of 0 ". 196 pixel $^{-1}$. The spacing between each array is $90 \%$ of an array

TABLE 1

LCIR Survey FIELDS

\begin{tabular}{|c|c|c|c|}
\hline Field & R.A. (2000) & Decl. (2000) & $\begin{array}{c}|b| \\
(\operatorname{deg})\end{array}$ \\
\hline NOAO-Cetus......... & 0210 & -0437 & 60 \\
\hline CDF-S.. & 0332 & -2738 & 54 \\
\hline NTT Deep .............. & 1205 & -0727 & 52 \\
\hline $1511 \ldots \ldots \ldots \ldots \ldots \ldots \ldots$ & 1524 & +0010 & 44 \\
\hline SSA $22 \ldots \ldots \ldots \ldots \ldots$ & 2200 & +0021 & 40 \\
\hline 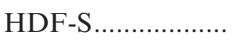 & 2233 & -6039 & 49 \\
\hline
\end{tabular}

NOTE.-Units of right ascension are hours and minutes, and units of declination are degrees and arcminutes. 
dimension. Therefore, one pass of four pointings $\sim 192^{\prime \prime}$ apart in a square pattern is needed to fill in the spaces between the arrays. A filled mosaic covers $\sim 13^{\prime} \times 13^{\prime}$. This defines our unit field area, hereafter referred to as a "tile."

Observations of individual pointings were composed of nine sets of three to five exposures 35-45 s in duration with dither offsets of between $8^{\prime \prime}$ and $13^{\prime \prime}$ in a rectangular pattern. For a typical $H$-band sky brightness of $14.7 \mathrm{mag}$, we estimated that a total exposure time of 80 minutes was needed to reach a $5 \sigma$ limiting magnitude of $H \sim 20.3$ in a $4^{\prime \prime}$ diameter aperture. The target integration time was achieved by making three passes on each field. Because it was often impossible to complete three passes of a field within one night, the observations of any given field area were spread over several nights. Standard stars from Persson et al. (1998) were observed three to five times per night. Flat-field observations were made using a screen hung inside the dome and were formed from differences of equal length exposures with the dome lamps on and off.

Most of our fields are composed of four contiguous tiles arranged to cover a roughly $25^{\prime} \times 25^{\prime}$ field. The HDF-S field contains seven tiles arranged in an $\mathrm{H}$-shaped pattern to avoid several very bright stars. The NTT Deep and 1511 fields contain tiles that are not contiguous. This was because the optical data were obtained with the sparse-array geometry Bernstein-Tyson Camera (BTC) on the $4 \mathrm{~m}$ telescope at CTIO. The journal of $H$-band imaging observations is given in Table 2, in which columns (1)-(5) and column (8) list the field, tile number, the 2000 coordinates of the tile centers, total exposure time, and date of observation, respectively.

\subsection{Optical Imaging Observations}

Optical images of the LCIR survey fields were obtained either from our own observations or from the literature using various large-format CCDs at different telescopes. The journal of optical imaging observations is given in Table 3, which lists the field, telescope, instrument, plate scale, field of view (FOV), bandpasses, total exposure time, and date of observation. A more detailed description of the optical imaging observations will be presented elsewhere (R. O. Marzke et al., in preparation).

\section{IMAGE PROCESSING}

In this section, we describe the procedures of processing the near-infrared $H$-band images of all six fields and summarize the qualities of the available optical images of the HDF-S and CDF-S regions. In addition, we discuss astrometric and photometric calibration of these images.

\subsection{The H-Band Images}

The $H$-band images obtained with CIRSI were processed using two independent reduction packages, one developed by McCarthy, Wilson, and Chen at Carnegie, the other by Lewis, Sabbey, McMahon, and Firth at the Institute of Astronomy (IoA).

The Carnegie reduction pipeline proceeds as follows. First, loop-combined images were formed by taking a mean of each loop of three to five exposures obtained without dithering the telescope. A $5 \sigma$ rejection algorithm was

TABLE 2

JournaL OF $H$-BAND IMAGING OBSERVATIONS

\begin{tabular}{|c|c|c|c|c|c|c|c|}
\hline $\begin{array}{l}\text { Field } \\
\text { (1) }\end{array}$ & $\begin{array}{l}\text { Tile } \\
(2)\end{array}$ & $\begin{array}{l}\text { R.A. (2000) } \\
\text { (3) }\end{array}$ & $\begin{array}{c}\text { Decl. (2000) } \\
\text { (4) }\end{array}$ & $\begin{array}{c}\text { Exposure } \\
\text { (s) } \\
\text { (5) }\end{array}$ & $\begin{array}{c}\text { FWHM } \\
\text { (arcsec) } \\
(6)\end{array}$ & $\begin{array}{l}H_{5 \sigma}{ }^{\mathrm{a}} \\
(7)\end{array}$ & $\begin{array}{l}\text { Date } \\
(8)\end{array}$ \\
\hline \multirow[t]{4}{*}{ NOAO-Cetus... } & 1 & 021013.0 & -043300.0 & 4800 & 0.80 & 20.1 & 1999 Oct $16-19$ \\
\hline & 2 & 020922.0 & -043300.0 & 2430 & 0.70 & 20.2 & 2000 Oct $09-09$ \\
\hline & 3 & 021013.0 & -044548.0 & 3300 & 0.72 & 20.2 & 2000 Oct $11-12$ \\
\hline & 4 & 020922.0 & -044548.0 & 2320 & 0.68 & 20.3 & 2000 Oct $09-09$ \\
\hline \multirow[t]{4}{*}{ CDF-S ................ } & 1 & 033236.0 & -274630.0 & 4420 & 0.66 & 21.3 & 2000 Oct $07-09$ \\
\hline & 2 & 033236.0 & -273353.0 & 4860 & 0.70 & 21.2 & 2000 Oct $05-07$ \\
\hline & 3 & 033139.0 & -273353.0 & 5535 & 0.69 & 21.2 & 2000 Sep $15-16$ \\
\hline & 4 & 033139.0 & -274630.0 & 6243 & 0.80 & 20.8 & 1999 Dec 20-31 \\
\hline \multirow[t]{4}{*}{ NTT Deep ........ } & 1 & 120557.8 & -074158.0 & 4700 & 0.80 & 20.5 & 1999 Feb $13-16$ \\
\hline & 2 & 120557.8 & -072152.0 & 4860 & 0.90 & 20.9 & 2000 Jan $01-17$ \\
\hline & 3 & 120436.6 & -074158.0 & 4691 & 0.80 & 20.6 & 2000 Feb $13-16$ \\
\hline & 4 & 120436.6 & -072152.0 & 5264 & 0.75 & 21.3 & 1999 Dec $26-30$ \\
\hline 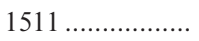 & 2 & 152407.7 & +002016.0 & 3600 & 0.68 & 20.1 & 2000 Feb $13-16$ \\
\hline \multirow[t]{4}{*}{ SA $22 \ldots \ldots \ldots \ldots$} & 1 & 221800.0 & +001434.0 & 4321 & 0.66 & 20.1 & 1999 Oct $16-24$ \\
\hline & 2 & 221708.0 & +001434.0 & 3510 & 0.60 & 20.8 & 2000 Oct $06-07$ \\
\hline & 3 & 221800.0 & +002730.0 & 4620 & 0.77 & 20.8 & 2000 Oct $04-06$ \\
\hline & 4 & 221708.0 & +002730.0 & 3731 & 0.65 & 20.5 & 2000 Oct $12-13$ \\
\hline \multirow[t]{7}{*}{ HDF-S. } & 1 & 223313.0 & -603927.0 & 4826 & 0.92 & 20.5 & 1999 Oct $18-19$ \\
\hline & 2 & 223456.0 & -603927.0 & 3645 & 0.90 & 20.5 & 1999 Oct $20-29$ \\
\hline & 3 & 223130.0 & -603927.0 & 4758 & 1.06 & 20.6 & 1999 Oct $25-29$ \\
\hline & 4 & 223456.0 & -602650.0 & 3645 & 0.84 & 21.3 & 2000 Sep 07-07 \\
\hline & 5 & 223456.0 & -604950.0 & 4230 & 1.00 & 21.1 & 2000 Sep $13-15$ \\
\hline & 6 & 223130.0 & -604950.0 & 6986 & 0.85 & 21.2 & 2000 Oct $04-11$ \\
\hline & 7 & 223130.0 & -602600.0 & 4890 & 0.85 & 21.3 & 2000 Oct $10-12$ \\
\hline
\end{tabular}

Note.-Units of right ascension are hours, minutes, and seconds, and units of declination are degrees, arcminutes, and arcseconds.

a The $5 \sigma$ detection threshold was determined over a $4^{\prime \prime}$ diameter aperture using a mean sky noise measured in each tile. The actual depth may vary, depending on the PSF, in each tile. 
TABLE 3

Summary of Optical Imaging Data

\begin{tabular}{|c|c|c|c|c|c|}
\hline Field & Telescope & Detector & $\begin{array}{c}\text { Plate Scale } \\
\left(\operatorname{arcsec~pixel~}^{-1}\right)\end{array}$ & $\begin{array}{c}\text { FOV } \\
\left(\operatorname{arcmin}^{2}\right)\end{array}$ & Bandpasses \\
\hline \multirow[t]{2}{*}{ NOAO-Cetus... } & CTIO $4 \mathrm{~m}$ & Mosaic II & 0.27 & $36 \times 36$ & $V$ \\
\hline & CFHT & CFH 12k & 0.20 & $40 \times 40$ & $R, I, z^{\prime}$ \\
\hline CDF-S ................. & CTIO $4 \mathrm{~m}$ & Mosaic II & 0.27 & $36 \times 36$ & $B, V, R, I, z^{\prime}$ \\
\hline \multirow[t]{2}{*}{ NTT Deep ........ } & CTIO $4 \mathrm{~m}$ & BTC & 0.43 & $29 \times 29$ & $V, R, I$ \\
\hline & CTIO $4 \mathrm{~m}$ & Mosaic II & 0.27 & $36 \times 36$ & $B, z^{\prime}$ \\
\hline \multirow[t]{2}{*}{$1511 \ldots \ldots \ldots \ldots \ldots$} & CTIO $4 \mathrm{~m}$ & BTC & 0.43 & $29 \times 29$ & $V, R, I$ \\
\hline & CTIO $4 \mathrm{~m}$ & Mosaic II & 0.27 & $36 \times 36$ & $B, z^{\prime}$ \\
\hline \multirow[t]{2}{*}{ SA22.. } & CTIO $4 \mathrm{~m}$ & Mosaic & 0.26 & $36 \times 36$ & $B, V, R, z^{\prime}$ \\
\hline & CFHT & CFH 12k & 0.20 & $40 \times 40$ & $I$ \\
\hline \multirow[t]{2}{*}{ 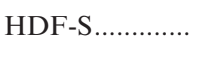 } & CTIO $4 \mathrm{~m}$ & BTC & 0.43 & $29 \times 29$ & $U, B, V, R, I^{\mathrm{a}}$ \\
\hline & $\mathrm{CTIO} / 4 \mathrm{~m}$ & Mosaic II & 0.27 & $36 \times 36$ & $z^{\prime}$ \\
\hline
\end{tabular}

a Teplitz et al. 2001.

applied to remove cosmic-ray events, if there were more than four exposures in a loop. Next, the loop-combined images were divided by the normalized dome flats and grouped together according to their locations on the sky. The use of a dome rather than dark-sky flat-field image allows us to separate the pixel-to-pixel gain variations from the additive sky fringes, which are nonnegligible for these Hawaii arrays. The flat-field images have been normalized to remove the gain differences between the four detectors. These are generally less than 5\%, except in the case of chip 3, for which the gain correction is $\sim 20 \%$. Next, a bad-pixel mask was generated for each night using the flat-field exposures.

Initial sky frames were constructed from medians of all frames for individual arrays that lay within a given pointing position. These sky frames were subtracted from each flatfield-corrected image for the purpose of constructing an object mask for each image. The sky-subtracted images were registered using the offsets derived from the centroids of 5-15 stars. All objects with peak intensities more than 10 $\sigma$ above the sky noise were identified and sorted into four categories according to their isophotal sizes. Mask frames were then generated for each dithered exposure accordingly. Next, a sliding-median sky frame was obtained by taking the median of the preceding and succeeding three to five images with the bad-pixel and object masks applied. After being scaled by the ratio of the modes in the images, the median sky frame was subtracted from the appropriate flatfield-corrected image to remove the fringes due to $\mathrm{OH}$ emission lines from the sky that are present in the flat-field-corrected images. This latter scaling (typically $\pm 1 \%$ ) allowed us to remove nonlinear temporal variations in the sky level. Finally, the flat-field-corrected and sky-subtracted images were registered and combined using a $5 \sigma$ rejection criterion, a bad pixel mask, and a weight proportional to the inverse of the sky variance to form a final stacked image for each pointing position.

The contiguous $13^{\prime} \times 13^{\prime}$ tiles were assembled from the 16 stacked images corresponding to the four arrays at four pointings of the telescope. The array boundaries were registered using stars in the overlap region, which was typically $20^{\prime \prime}$ to $30^{\prime \prime}$ in extent. The four arrays were not perfectly aligned with each other and a significant rotation $\left(\sim 0^{\circ} .4\right)$ was found necessary to bring one of the arrays into registration with the other three. The final image was constructed by summing the 16 registered stacked images. A $5 \sigma$ rejection criterion and a weighting determined from the sky variance were also applied in the vicinity of the array boundaries. A $1 \sigma$ error image was formed simultaneously for each tile through appropriate error propagation, assuming Poisson counting statistics.

The IoA-based reduction pipeline is similar in conceptual design, although it is written in a somewhat different architecture and contains a few operational differences (Sabbey et al. 2001). The offsets between dithered images were derived via cross-correlations in the IoA pipeline rather than from image centroiding. The object masks were derived from segmentation maps produced by SExtractor (Bertin \& Arnout 1996) rather than using a set of five fixed object sizes as in the Carnegie pipeline. Finally, the assembly of the final tiles was completed after the application of a world coordinate system derived from comparison with the APM catalog. In the Carnegie pipeline the astrometry was derived for the completed tiles using the USNO astrometric catalog, discussed in $\S 3.3$.

The images of the first three tiles of the HDF-S region, the first tile of the NTT field, and the second tile of the 1511 field contained significant artifacts associated with instabilities in the detector reset. These manifest themselves as low spatial frequency bias roll-offs that change shape and amplitude on short timescales. Both the Carnegie and IoA pipelines deal with this low-frequency signal by fitting low-order polynomials to $\sigma$-clipped images on a line-by-line and column-by-column basis. In the Carnegie pipeline this reset correction is applied after the final sky subtraction; in the IoA pipeline it is applied before the flat-field and sky corrections have been determined. New readout electronics, which largely suppress the reset instability, were implemented in 2000 August.

The full width at half-maximum (FWHM) of a typical point-spread function (PSF) and the $5 \sigma$ detection threshold in a $4^{\prime \prime}$ diameter aperture of each completed tile are given in columns (6) and (7) of Table 2, respectively. The PSF was measured to vary by no more than $10 \%$ across a tile, but the $5 \sigma$ sky noise was found to vary by as much as $50 \%$ across each tile. Figure 2 shows the distributions of the FWHM of a mean PSF (left) and the $5 \sigma$ detection threshold estimated based on mean sky noise in a $4^{\prime \prime}$ diameter aperture (right) for each completed tile. Figure 3 shows an example of a completed tile in the CDF-S region from the Carnegie pipe- 

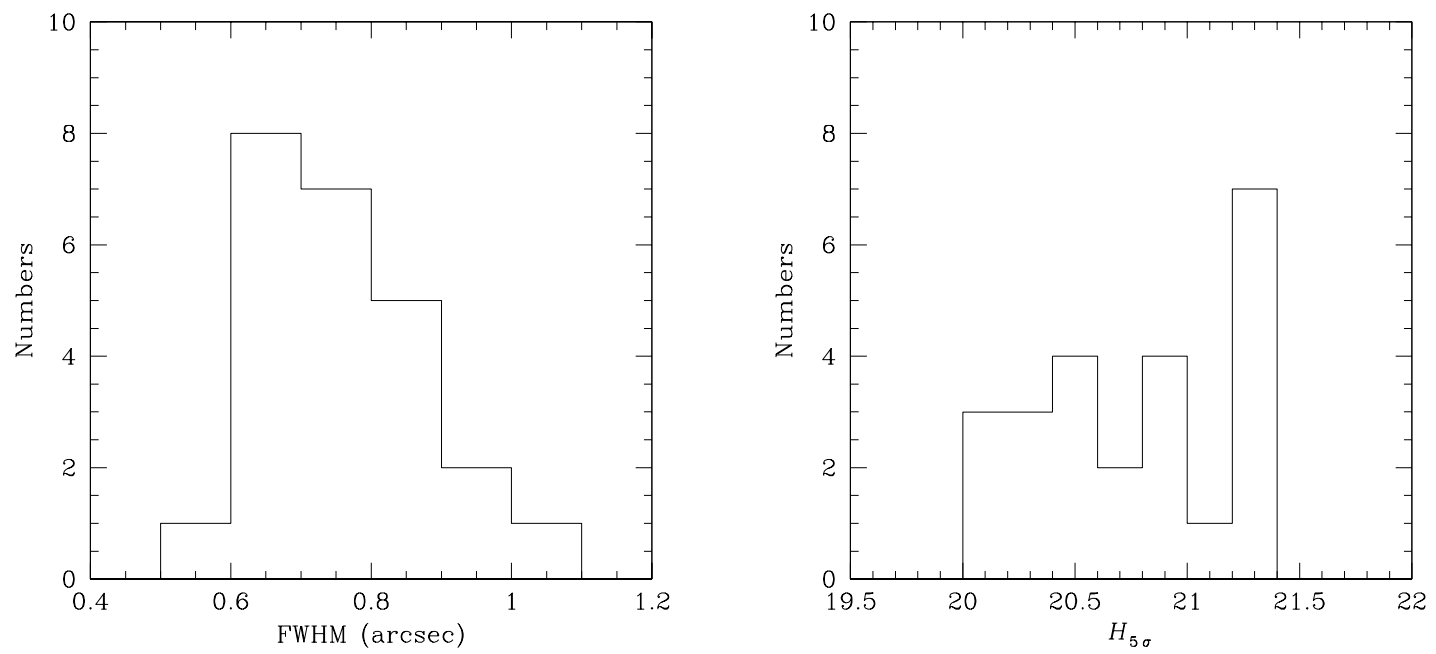

FIG. 2.- Summaries of the image quality of the $H$-band survey. The left panel shows the distribution of the FWHM, in arcseconds, of a mean PSF in each tile. The right panel shows the distribution of the $5 \sigma H$-band detection threshold in each tile estimated based on mean sky noise over a $4^{\prime \prime}$ diameter aperture.

line. The average PSF of this image has a FWHM of $\approx 0$ !"7. The magnitudes of the two objects indicated by arrows are $H=19.4$ and 21.3 .

\subsection{The Optical Images}

The optical $U, B, V, R$, and $I$ images of an $40^{\prime} \times 40^{\prime}$ area around the HDF-S region were obtained and processed by a team at the Goddard Space Flight Center during the HDF-S campaign using the BTC (Teplitz et al. 2001). The spatial resolutions of the final combined images were measured to range from FWHM $\approx 1$ ". 4 in the $V$ band to $\approx 1$ ". 6 in the $B$ band, and the $5 \sigma$ detection thresholds in a $4^{\prime \prime}$ diameter aperture were measured to be $U \sim 24.6, B \sim 26.1$, $V \sim 25.6, R \sim 25.1$, and $I \sim 24.2$ mag using the photometric zero points determined by the Goddard team.

The optical $V, R, I$, and $z^{\prime}$ images of $\approx 36^{\prime} \times 36^{\prime}$ around the CDF-S region were obtained using the Mosaic II imager on the $4 \mathrm{~m}$ telescope at CTIO in 1999 November. Individual images were processed following the standard mosaic image-reduction procedures for data obtained with the Mosaic II imager, registered to a common origin, and coadded to form final combined images (R. O. Marzke et al., in preparation). The spatial resolutions of the final combined images were measured to range from FWHM $\sim 1$ ". 0 in the $I$ and $z^{\prime}$ bands to $\approx 1^{\prime \prime} 2$ in the $V$ band, and the $5 \sigma$ detection thresholds in a $4^{\prime \prime}$ diameter aperture were measured to be $V \sim 26.8, R \sim 26.2, I \sim 25.3$, and $z^{\prime} \sim 23.7$ mag.

\subsection{Astrometry}

Accurate astrometry is required for follow-up observations and for matching our catalog with observations at other wavelengths. The optical images of the HDF-S region were corrected for astrometric distortions with an rms error of 0.16 pixels, corresponding to 0 ".07 (Teplitz et al. 2001). We adopted the World Coordinate System (WCS) solution stored in the image headers to derive coordinates for objects identified in the images. To correct astrometric distortions for the Mosaic II images of the CDF-S region, we first resampled images of individual exposures to a Cartesian grid before co-addition and derived a best-fit, fourth-order polynomial for the WCS solution by matching stars in the US Naval Observatory catalog (USNO-A2.0) with stars identified in the images (R. O. Marzke et al., in preparation). A total of 200 stars were included to obtain the best-fit astrometric solution. The goodness of fit can be characterized by an $\mathrm{rms}$ residual of $\approx 0$ ". 2 .

Astrometric solutions were also derived for the nearinfrared images. We were able to identify on average $\sim 150$ unblended and unsaturated stars in each $H$-band tile. Using a third-order polynomial fit we were able to obtain solutions with rms residuals of between 0 ".25 and 0 ". 35 .

\section{ANALYSIS}

In this section, we describe the procedures for object detection in individual bandpasses and object matching between frames taken through the various filters. We also examine the completeness limits of our detection algorithm and the reliability of photometric measurements in all bandpasses.

\subsection{Object Detection}

To obtain a uniform sample of distant galaxies of all types, it is crucial to identify galaxies of a wide range of optical and near-infrared colors. To reach this goal, we performed object detection using SExtractor for each bandpass in the regions where the $H$-band imaging survey was carried out. To reliably identify all the faint objects in each of the optical images, we set the SExtractor parameters by requiring that no detections be found in the negative image. First, we set the minimum area according to the FWHM of a mean PSF. Next, we adjusted the detection threshold to the lowest value that is consistent with zero detections in the negative image. To aid in deblending overlapping objects, we first constructed a "white light" image by co-adding all the registered optical images that had been scaled to unit exposure time and filter throughput (see the next section for descriptions of image registration). Next, we applied SExtractor to the white-light image and determined object extents based on their instrumental colors. Because the PSF varied between different optical bandpasses by at most $10 \%$ in each field, we were able to reliably deblend overlapping 


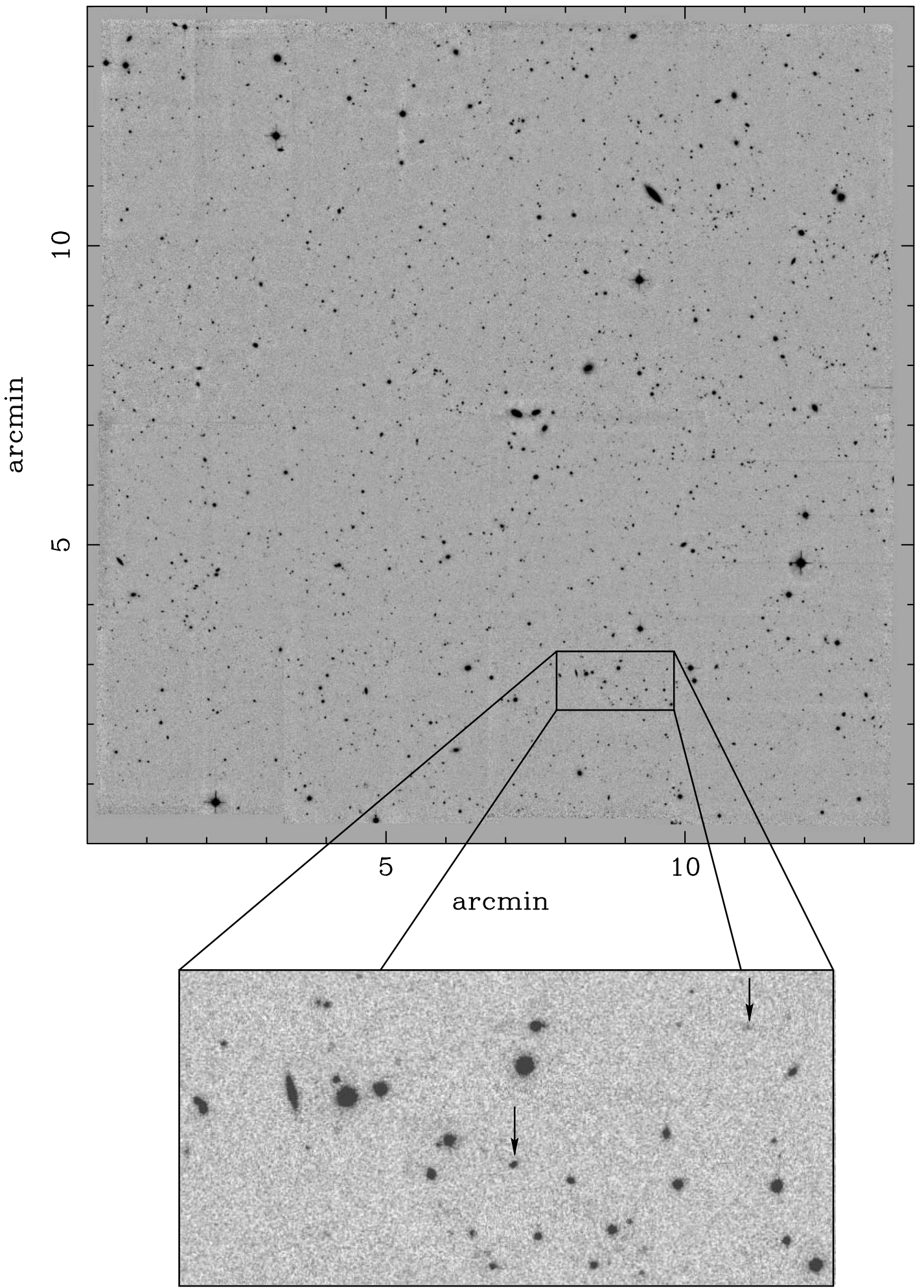

FIG. 3.-Example of an assembled tile in the CDF-S region. The image is approximately 13.5 on a side. The $5 \sigma$ point-source detection limit is $H \approx 20.9$ in a $4 "$ diameter aperture in this tile, and the PSF is $\approx 0$ " 7 FWHM. The bottom panel shows details of an $\approx 2 \times 1 \operatorname{arcmin}^{2}$ region. To illustrate the depth and contrast of the image, we have selected two objects of $H=19.4$ (left) and 21.3 (right), respectively, as indicated by the arrows.

objects without erroneously splitting or blending individual objects in the white-light image due to a large variation in the PSFs of different bandpasses. This procedure takes into account the intensity contrast between overlapping objects in all bandpasses instead of a single bandpass, allowing a more accurate object deblending for overlapping objects with different instrumental colors.

We adopted a different approach to reliably identify all the faint objects in the $H$-band images. Because of a nonuniform noise pattern between individual arrays across a single 
tile, in particular in the join regions between adjacent array images, the zero negative detection criterion would force a significant underestimate of the survey depth in some areas. An example to illustrate the noise pattern is given in Figure 4 , which shows the associated $1 \sigma$ noise image of the tile shown in Figure 3. We identified objects well into the noise in the $H$-band images and used our subsequent calibration of the completeness limits and photometric errors to set the faint magnitude limit of the $H$-band catalog.

Finally, to ensure accurate measurements of optical and near-infrared colors, we excluded objects that lie within $\approx 2^{\prime \prime}$ of the edges of each tile.

\subsection{Image Registration and Catalog Matching}

The optical $U, B, V, R$, and $I$ images of the HDF-S region were registered to a common origin by the Goddard team. The rms dispersion in the object coordinates between different bandpasses was measured to be within $\approx 0.2$ BTC pixels (corresponding to $0 . \prime 08$ ). The optical $V, R, I$, and $z^{\prime}$ images of the CDF-S region were registered individually for each area covered by a near-infrared tile, based on a solution determined using $\sim 200$ common stars identified in the tile. We applied standard routines to obtain the transformation solutions and to transform the images. We were able to register the optical images of the CDF-S tiles by a simple transformation of low-order polynomials in the $x$ - and $y$ directions without significantly degrading the image quality. To assess image degradation after the transformation, we measured the changes in the FWHM of the PSFs in transformed images and found an increase of no more than $5 \%$. The rms dispersion in the object coordinates between different bandpasses across an area spanned by a tile was measured to be within $\sim 0.3$ Mosaic II pixels (corresponding to 0. . 08 ). The dispersion was found to be the worst (as much as 0.5 Mosaic II pixels or 0"13) in the join area between adjacent arrays of the Mosaic II imager. To merge individual optical catalogs of each field, we stepped through each object identified in the white-light image, examined the presence/absence of object detections at the location in individ-

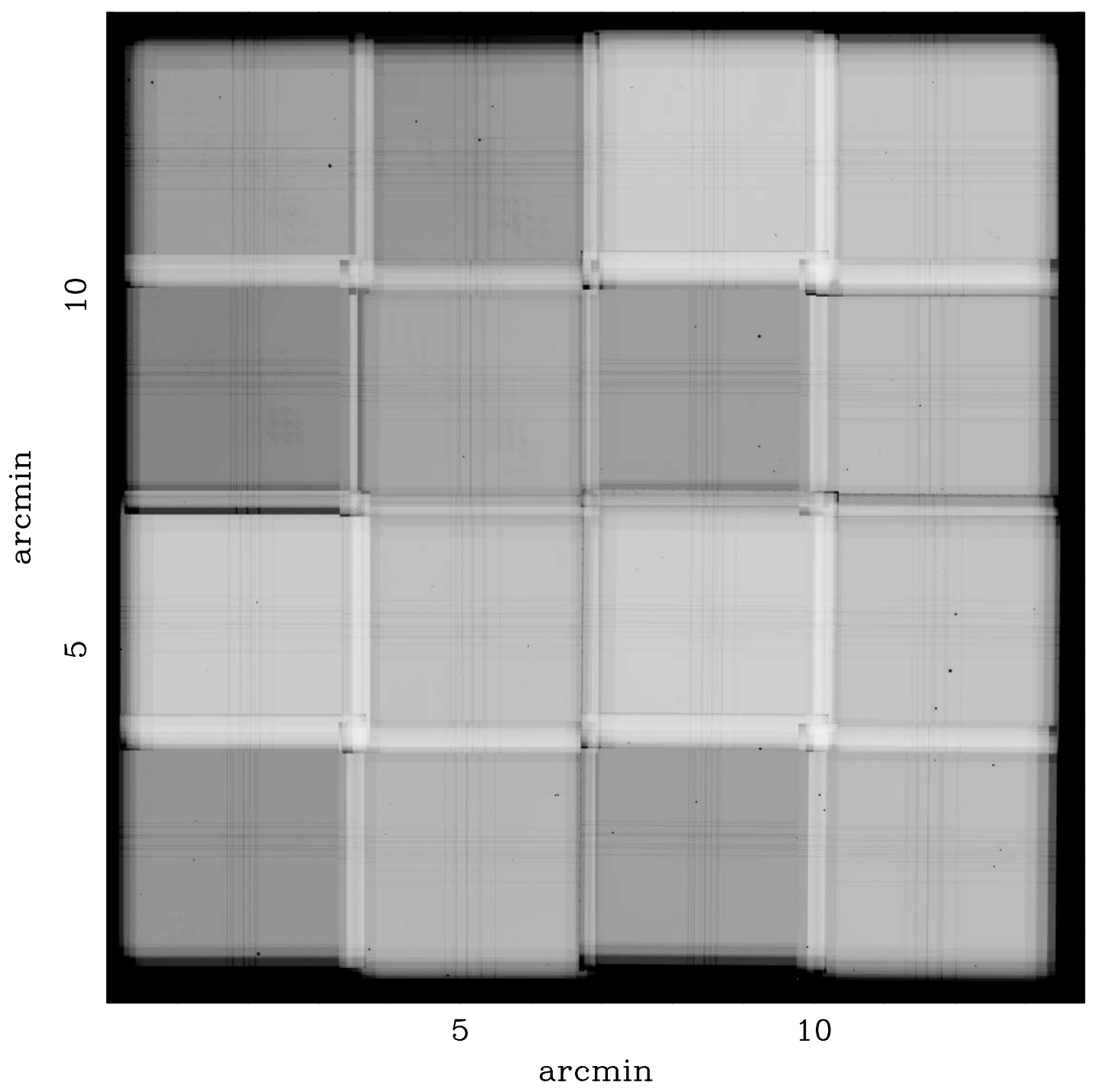

Fig. 4.-Associated $1 \sigma$ noise image of Fig. 3. The image dimension is the same as Fig. 3. Darker regions represent nosier areas. The composition of 16 stacked images, corresponding to four arrays at four pointings of the telescope, is clearly illustrated by the "square" pattern in the image. The noise level varies between arrays and pointings because of the differences in array sensitivity and total exposure time. Black dots indicate the locations of bright stars, where the noise level is dominated by object flux instead of sky. 
ual bandpasses, and recorded the SExtractor measurements for detections, placing null values for no detections in the combined optical catalog.

The $H$-band and optical catalogs were merged on the basis of astrometric position matching, rather than by transforming the $H$ images onto the same pixels as the optical images. The large differences in pixel scales and in the PSFs of the optical and IR images result in significant image degradation if one transforms the $H$ images to match the optical images. We derived a mapping between the $H$ and optical coordinates from $\sim 150$ common stars in each tile. The rms dispersion between the transformed optical coordinates and the $H$-band coordinates was measured to be $\approx 0.2$ CIRSI pixels (corresponding to 0".04). Next, we repeated the catalog merging procedure described above by examining the presence/absence of object detections within a radius of two CIRSI pixels to the transformed object location in the $H$-band image for all optically detected objects. The combined catalog was updated accordingly throughout the procedure. Finally, we compared objects in the combined optical catalog with those in the $H$-band catalog, identified those that appeared only in the $H$-band catalog, and included them to form the final combined optical and near-infrared catalog for each tile.

\subsection{Survey Completeness}

Since detection efficiencies vary significantly for objects of different intrinsic profiles because of the strong surface brightness selection biases and because the noise patterns of the $H$-band images vary across individual tiles, particularly at the edges of individual arrays, it is crucial to understand the sensitivities and detection efficiencies of the LCIR survey in order to ensure the reliability of future analyses. To understand the completeness limits of the $H$-band images (similar analyses for the optical images will be presented in R. O. Marzke et al., in preparation), we performed a series of simulations, the results of which were also adopted for testing the photometric accuracy described in the next section. First, we generated test objects of different brightness for various PSF-convolved model surface brightness profiles. We adopted a Moffat (1969) profile to simulate the PSFs and considered (1) a point source model, (2) a de Vaucouleurs' $r^{1 / 4}$ model characterized by a half-light radius $r_{e}=0.3,{ }^{9}$ and (3) three exponential disk models characterized by scale radii of $r_{s}=0$ " 15,0 ". 3 , and 0 ". 6 , respectively. The upper boundary of the selected disk scale lengths was determined according to the results of Schade et al. (1996) based on galaxies identified in the CNOC survey. These authors found a mean scale length $r_{s} \sim 0$ ". 6 for field galaxies at $z \sim 0.5$. Next, we placed the test objects of a given magnitude at 1000 random positions in an $H$ tile and repeated the object detection procedures using the same SExtractor parameters. Finally, we measured the recovery rate of the test objects as a function of $H$-band magnitude.

The results of the completeness test are presented in Figure 5 for tile 1 of the CDF-S field. Figure 5 shows that the $50 \%$ completeness limit of the image is $\sim 0.6 \mathrm{mag}$ fainter for an exponential disk of $r_{s}=0.13$ than for the largest disk model of $r_{s}=0$ ". 6 and is an additional $\sim 0.6$ mag fainter for a point source. The large difference between the completeness limits for objects of different intrinsic profiles makes an

\footnotetext{
$91^{\prime \prime}$ corresponds to a projected proper distance of $5.6 h^{-1} \mathrm{kpc}$ at $z=1$.
}

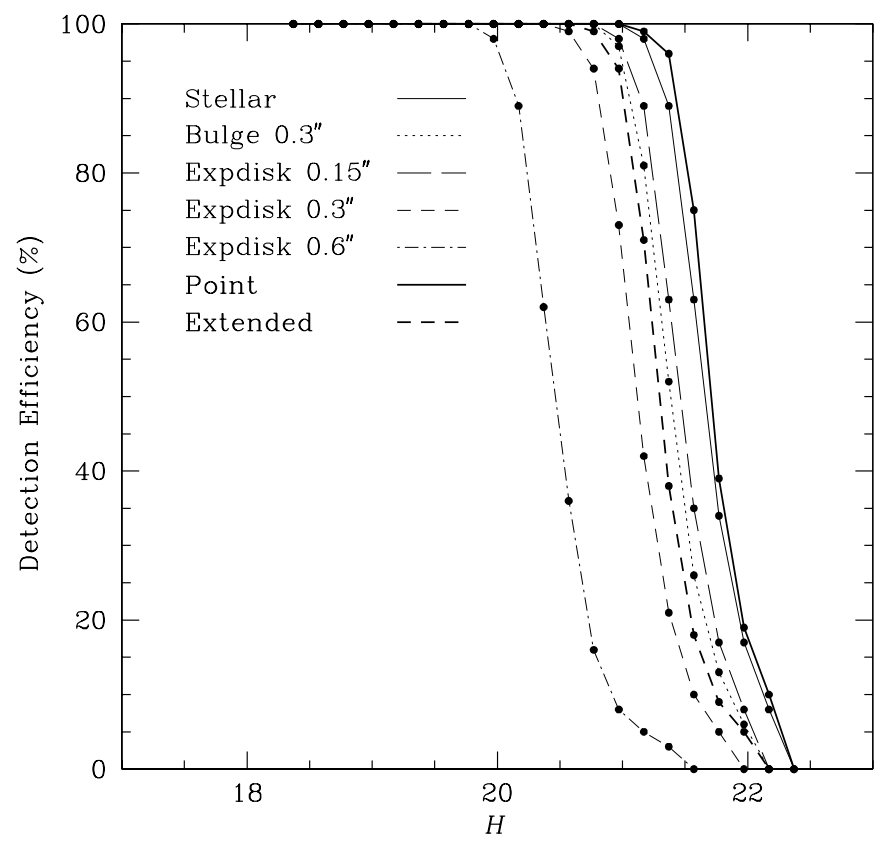

FIG. 5.-Detection efficiency estimated in one of the CDF-S $H$ tiles for objects of different intrinsic profiles and angular sizes. As indicated in the upper left corner, the solid curve represents a stellar profile; the dotted curve represents an $r^{1 / 4}$ de Vaucouleur profile with a half-light radius of 0". 3; the long-dashed, short-dashed, and dash-dotted curves represent disk profiles with scale radii of $0.15,0$ " 3 , and 0 ". 6 , respectively. The thick curves show the detection efficiencies for a point-source profile (solid curve) and an extended profile (short-dashed curve) determined empirically by co-adding individual object images (see $\S 4.3$ for a detailed description).

accurate incompleteness correction of the survey very challenging, because in practice we have no prior knowledge of the intrinsic profile of each source. It appears that under these circumstances correcting for the incompleteness based on one of these curves becomes arbitrary and therefore less accurate.

We repeated the simulations based on empirical surface brightness profiles determined from the image itself to address this problem and obtain a more meaningful estimate of the survey completeness. It is clear in Figure 5 that the completeness of an image is in practice set by the limit at which we can identify the majority of extended sources and that the survey for the largest disks in the image becomes incomplete at $H$ at least $1.5 \mathrm{mag}$ brighter than the $50 \%$ completeness limit for point sources. First, we adopted the stellarity index estimated by SExtractor, which according to Bertin \& Arnouts (1996) is nearly unity for a point source and zero for an extended object. We identified objects of stellarity $\gtrsim 0.95$ and with an $H$-band magnitude between $H=17$ and 18 , and co-added the individual images of these compact sources to form a high signal-to-noise ratio empirical profile for point sources. Next, we repeated the simulations using the empirical profile to construct a completeness curve as a function of $H$ for point sources. The results are shown by the thick solid curve in Figure 5 . Next, we determined the $50 \%$ completeness limit $H_{50}$ for point sources and identified extended sources of stellarity $\lesssim 0.5$ and with an $H$-band magnitude between $H=H_{50}-2$ and $H_{50}-1.5$ (to include a large enough number of objects to form a smooth image). We co-added the individual images of these extended sources to form a high signal-to-noise ratio empirical profile for extended sources. Finally, we repeated the 
simulations using the empirical profile to construct a completeness curve as a function of $H$ for extended sources. The results are shown as the thick dashed curve in Figure 5. The $95 \%$ completeness is $\approx 0.5 \mathrm{mag}$ shallower for extended sources than for point sources. The completeness curve for the empirical profile of an extended source falls between those of $r_{s}=0$ ". 15 and $0 . " 3$, suggesting that sources with a large extended disk do not constitute a large portion of the faint population. We repeated this procedure for all $H$ tiles.

\subsection{Photometric Accuracy}

Photometric calibrations of the optical $U, B, V, R$, and $I$ images were obtained by fitting a linear function, which included a zero-point and color and extinction corrections, to the Landolt standard stars (Landolt 1992) observed on the same nights. Photometric calibrations of the $z^{\prime}$ images were obtained using the Sloan Digital Sky Survey secondary standards provided in Stoughton et al. (2002), who adopted the AB magnitude system. We converted the best-fit zero point to the Vega magnitude system, using $z_{\mathrm{AB}}^{\prime}=z^{\prime}+0.55$ (Fukugita, Shimasaku, \& Ichikawa 1995), for calibrating photometry of our objects. The rms dispersion of the bestfit photometric solution was found to be $\lesssim 0.03 \mathrm{mag}$ in all optical images of the CDF-S field (a detailed discussion will be presented in R. O. Marzke et al., in preparation). Photometric calibrations of the $H$-band images were obtained by fitting a constant term that accounts for the zero-point correction to a number of faint near-infrared standard stars (Persson et al. 1998) observed throughout every night. The rms dispersion of the best-fit photometric solution was found to be $\lesssim 0.02 \mathrm{mag}$. The zero point was found to remain stable between different nights with a scatter of $\lesssim 0.04$ mag.

To cross-check the photometric accuracy of objects identified in the HDF-S and CDF-S regions, we first compared our photometry with that from the EIS project (da Costa et al. 2001) on an object-by-object basis. This clearly revealed an offset in the $I$-band photometry for the HDF-S. Next, we compared optical colors of bright, compact sources (which are likely to be stars) with the UBVRI colors of subdwarfs presented in Ryan (1989). We found that the stellar track agrees well with Ryan's measurements in all colors for objects in the CDF-S region, but not for objects in the HDF-S region in the $U, V$, and $I$ bandpasses. Based on the results of stellar track comparison, we concluded that adjustments of $U=U_{\text {Goddard }}+0.3, \quad V=V_{\text {Goddard }}+0.1$, and $I=I_{\text {Goddard }}+0.27$ may be needed in order to bring the stellar colors into agreement, where $U_{\text {Goddard }}, V_{\text {Goddard }}$, and $I_{\text {Goddard }}$ are the photometric zero points published by the Goddard team. ${ }^{10}$ This also brought our photometry very close to the EIS photometry of the same objects in the HDF-S region.

Accurate photometry of faint galaxies is hampered by the unknown intrinsic surface brightness profiles of the galaxies and by strong surface brightness selection biases. Accurate total flux measurements of distant galaxies are crucial not only for an accurate assessment of galaxy evolution in luminosity function (e.g., Dalcanton 1998) but also for comparing the observed number counts with theoretical predictions based on different cosmological models. Various approaches to measuring the total fluxes of faint galaxies have been proposed. For example, an "adapted" aperture

${ }^{10}$ Available at http://hires.gsfc.nasa.gov./ research/hdfs-btc. magnitude, also known as a Kron magnitude, measures the integrated flux within a radius that corresponds to the first moment of the surface brightness distribution of an object and corrects for the missing flux outside the adopted aperture, which was found to be a constant fraction of the total flux for objects of different surface brightness profiles (Kron 1980). A simpler way is to measure the integrated flux within a fixed aperture and correct for the missing flux using a growth curve derived from the PSF of the image.

To understand how well various approaches are able to recover the total flux for objects identified in the survey, we performed a series of photometric tests for objects of different brightness and intrinsic surface brightness profiles based on the same simulations as described in $\S 4.3$. First, we generated test objects of different brightness using a series of PSF-convolved model surface brightness profiles. Next, we placed the test objects at 1000 random positions in an $H$ tile and ran SExtractor on the simulated object image using the same photometric parameters. Finally, we compared various photometric measurements with the model fluxes.

We present in Figure 6 comparisons of model fluxes and various photometric measurements for objects of different intrinsic surface brightness profiles to the $90 \%$ completeness limit of the image. The leftmost panel shows the test results for corrected isophotal magnitudes provided by SExtractor, the center panel for aperture magnitudes within a $4^{\prime \prime}$ diameter, and the right panel for the " best " magnitudes estimated by SExtractor, which are either Kron magnitudes or corrected isophotal magnitudes in cases of crowded areas. The plotted points are the median residuals, and the error bars indicate the 16th and 84th percentiles of the residuals in 1000 realizations. Three interesting points are illustrated in Figure 6. First, the corrected isophotal magnitudes always underestimate the total flux at faint magnitudes, irrespective of object intrinsic surface brightness profile. Second, the 4 " diameter aperture photometry after a small, fixed aperture correction appears to deliver accurate total flux measurements for all but the largest disk model at all magnitudes to the $90 \%$ completeness limits. Third, the SExtractor "best" magnitudes appear to be able to recover the total flux of every profile except for the largest disks at the faintest magnitudes, although it also appears that a small amount of "aperture" correction is needed to bring the centroids of the residuals to zero. Because galaxies of scale length greater than 0 ". 5 , corresponding to 0 " 8 half-light radius, appear to be rare (e.g., Yan et al. 1998), we conclude that the corrected $4^{\prime \prime}$ aperture magnitude provides the most accurate estimate of the total object flux. Although the use of a relatively large aperture results in reduced signal-to-noise ratios and hence a loss in depth, the loss in precision is offset by the improved accuracy in both the total magnitude measurements and the determination of colors from images with a wide range of seeing.

\subsection{Star and Galaxy Separation}

Stellar contamination of the final galaxy catalog from the LCIR survey is nonnegligible, particularly in the HDF-S. An accurate separation of stars from the galaxy catalog is therefore essential for subsequent statistical analysis. To identify stars in the survey fields, either we can rely on morphological classification by comparing object sizes and shapes with the PSF, or we can classify objects on the basis of their spectral shapes. Neither approach by itself can robustly identify stars at all signal-to-noise ratio levels. 


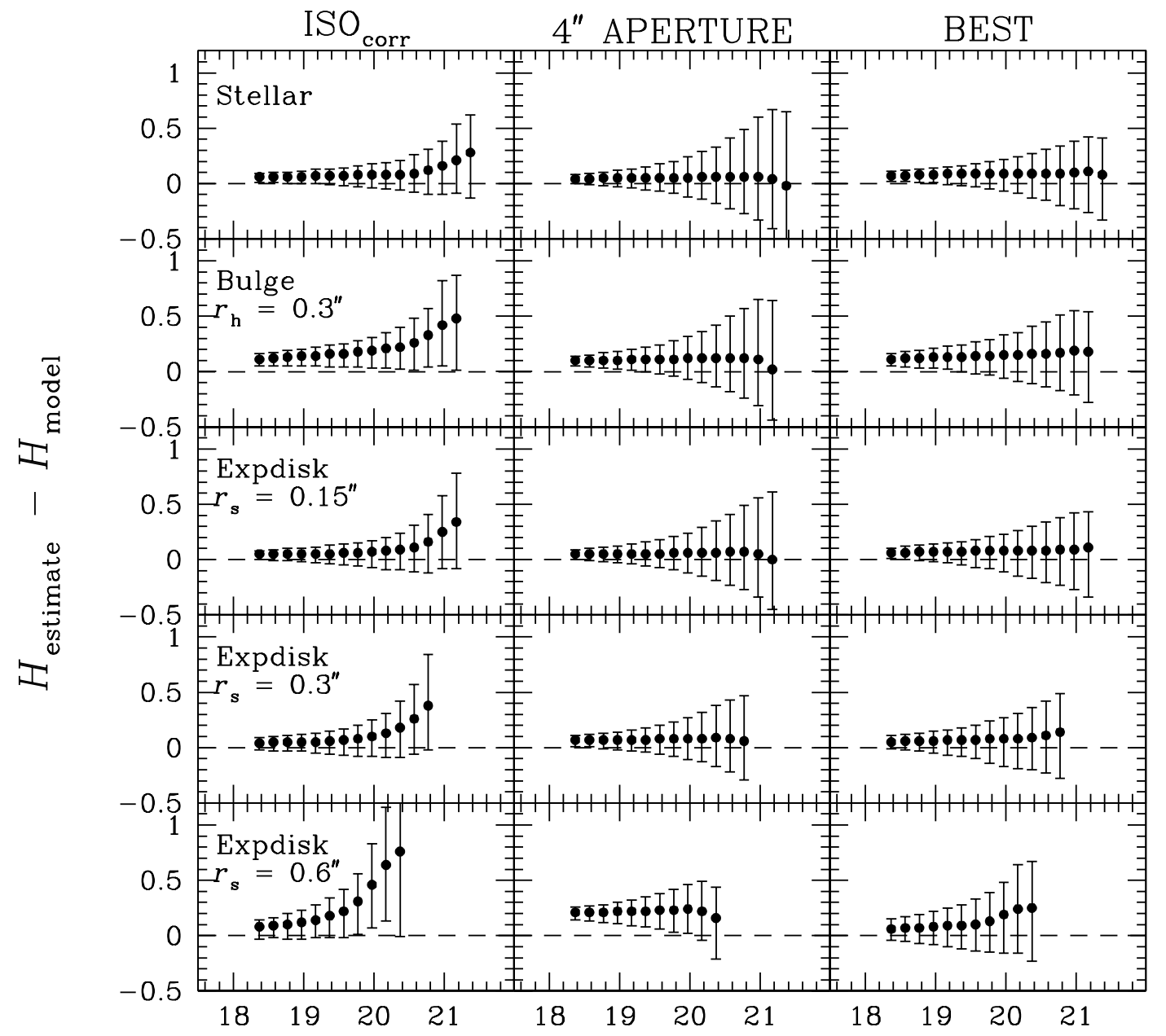

$H$

FIG. 6. - Comparisons of model fluxes and various photometric measurements for objects of different brightness and intrinsic surface brightness profiles. The first column shows the test results for corrected isophotal magnitudes provided by SExtractor, the second column for aperture magnitudes within a $4^{\prime \prime}$ diameter, and the third column for the " best" magnitudes estimated by SExtractor. Filled circles show the median residuals, and error bars indicate the 16th and 84 th percentile of the residuals in 1000 realizations. The last circle in each panel corresponds to the $90 \%$ completeness limit of the image, which varies with object intrinsic surface brightness profile.

To test the reliability of the stellarity index provided in SExtractor at different signal levels, we measured the rate at which a point source has a measured stellarity index less than a threshold value in the completeness test described in $\S 4.3$. The results are shown in Figure 7. The solid curve indicates the fraction of the input stars that have a stellarity index $\lesssim 0.5$ as a function of $H$-band magnitude. The dashed curve indicates the fraction of stars that have a stellarity index $\lesssim 0.8$. The dotted line indicates the $50 \%$ completeness limit $\left(H_{50}\right)$ of the test image for a point source. We found that while SExtractor is able to uncover point sources at fairly bright magnitudes, it becomes unreliable at around $H_{50}-2$ mag (i.e., more than $20 \%$ of stars would be misclassified as galaxies) and unstable at around $H_{50}-1.5 \mathrm{mag}$ (where objects are more or less equally divided between compact and extended sources irrespective of their true shapes), and therefore cannot by themselves be adopted to robustly separate stars from galaxies. In addition, identifying stars based on morphological classification alone introduces biases against compact sources such as quasars, narrow emission line galaxies, etc.
To test the reliability of comparing object colors with known stellar energy distributions, we first included a suite of empirical stellar templates collected from the literature (Oppenheimer et al. 1998; Pickles 1998; Fan et al. 2000; Leggett et al. 2000) in the photometric redshift likelihood analysis (H.-W. Chen et al., in preparation). The adopted stellar templates span a wide range in both spectral coverage, from optical to near-infrared wavelengths, and stellar types, from early-type $\mathrm{O}$ and $\mathrm{B}$ stars to late-type $\mathrm{L}$ and $\mathrm{T}$ dwarfs. Then we compared the results of photometric redshift analysis with spectroscopic redshift measurements collected from the literature for objects in the HDF-S region. We found that while all the spectroscopically identified stars were accurately identified as stars based on the photometric redshift analysis, $\sim 10 \%$ of the spectroscopically identified galaxies were misidentified as stars. Therefore, we conclude that photometric redshift analysis with our present filter set alone cannot robustly separate galaxies from stars.

To improve the accuracy of star and galaxy separation, we investigated a method of combining both approaches. We first examined the stellarity index estimated for each 


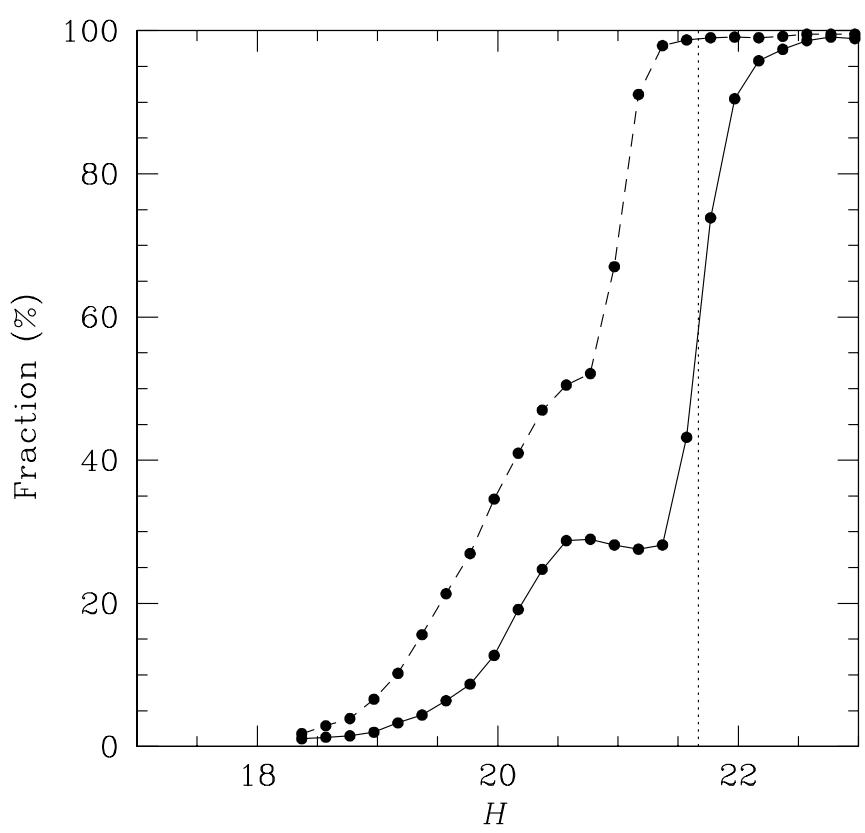

FIG. 7.- Robustness of using the stellarity index provided in SExtractor to identify stars. According to Bertin \& Arnouts (1996), a point source has a stellarity index close to 1 , while an extended object has a stellarity index close to zero. The solid curve indicates the fraction of the input stars that have a stellarity index $\lesssim 0.5$ as a function of $H$-band magnitude. The dashed curve indicates the fraction of stars that have a stellarity index $\leq 0.8$. The dotted line indicates the $50 \%$ completeness limit of the test image for a point source.

object by SExtractor in all bandpasses. For objects with a stellarity index greater than 0.95 in one or more bandpasses, we used both stellar and galaxy templates in the photometric redshift analysis. For objects with a stellarity index less than 0.95 in all bandpasses, we used only galaxy templates in the photometric redshift analysis. Comparison of photometric and spectroscopic redshifts for objects in the HDF-S region demonstrates that all 11 spectroscopically identified stars can be reliably identified in this procedure.

The addition of other colors (e.g., $J$ and $K$ ) will improve the photometric separation of stars and galaxies. At present, the star and galaxy separation may be inaccurate at faint magnitudes, where the stellarity index becomes unreliable. However, galaxies are likely to dominate the number counts at faint magnitudes (see $\S 6.2$ ). Figure 8 shows the stellar fraction in the HDF-S and CDF-S regions based on the results of the star and galaxy separation. Filled circles indicate the mean surface density of galaxies observed in the two fields (see $\S 6.2$ for a detailed discussion); stars indicate the surface density of stellar objects in the HDF-S region; and open triangles indicate the surface density of stellar objects in the CDF-S region. It shows that the star counts increase gradually with the $H$ magnitude, while the galaxy counts show a much steeper slope. The smooth, shallower slope of the star counts suggests that stars do not contribute a significant fraction at $H \gtrsim 19$ in either the HDF-S or CDF-S fields.

\section{THE CATALOGS}

The $H$-band imaging survey presently covers $1.1 \mathrm{deg}^{2}$ of sky to a mean $5 \sigma$ detection limit in a $4^{\prime \prime}$ diameter aperture of $H \sim 20.8$. Here we present sample near-infrared and optical

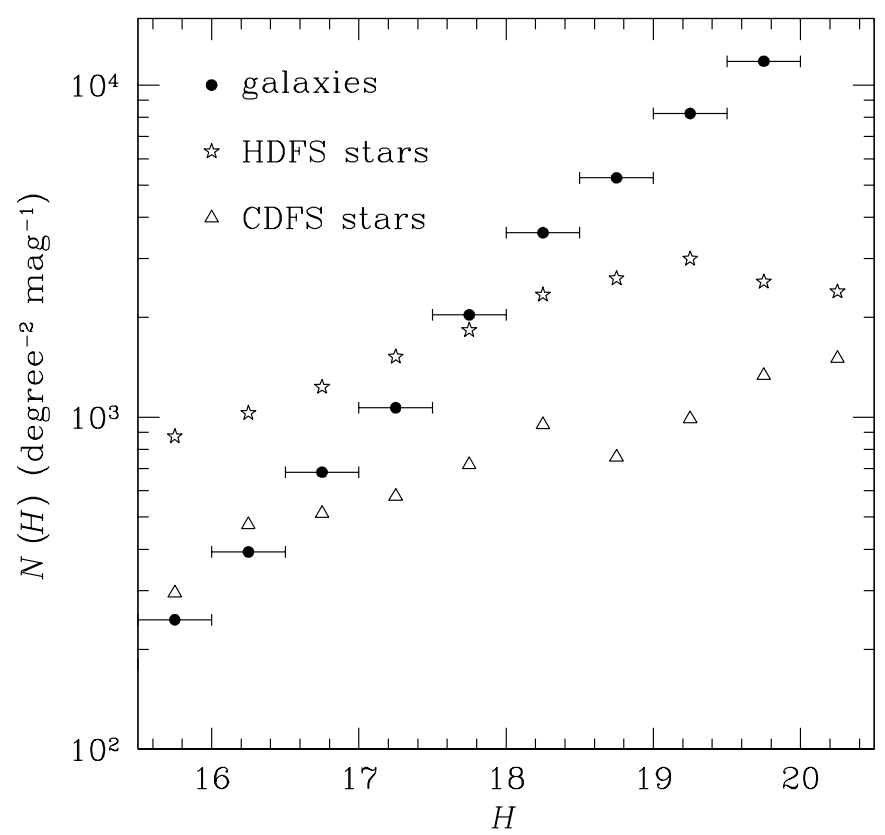

FIG. 8. - Stellar fraction in the HDF-S and CDF-S regions. Filled circles indicate the mean surface density of galaxies observed in the two fields; stars indicate the surface density of stars in the HDF-S region; open triangles indicate the surface density of stars in the CDF-S region.

photometric measurements for objects identified in the HDF-S and CDF-S regions. We have performed object detection in all bandpasses and identified $\sim 24,000$ galaxies over $847 \operatorname{arcmin}^{2}$ in the HDF-S region, of which $\sim 6700$ are detected in the $H$-band survey, and $\sim 30,000$ galaxies over $561 \operatorname{arcmin}^{2}$ in the CDF-S region, of which $\sim 7400$ are detected in the $H$-band survey. Among the $H$-detected galaxies in the two regions, $\sim 2000$ have $I-H>3$ and are likely to be primarily evolved galaxies at redshifts $z>1$.

The catalogs for each field are organized as follows. For each object we give an identification number, $x$ and $y$ pixel coordinates in the optical images, $\alpha$ (J2000) and $\delta$ (J2000), the Kron radius (in arcseconds) for each filter, the stellarity index determined by SExtractor, $2^{\prime \prime}$ and $4^{\prime \prime}$ diameter aperture magnitudes and their associated uncertainties, and the corrected isophotal magnitude, auto magnitude, and the best magnitude and their associated uncertainties as provided by SExtractor for all the objects detected in each filter. We present an example of the object catalog in Table 4 and an example of the photometric catalog in Table 5, which list the first 50 objects identified in the CDF-S region. Complete near-infrared and optical photometric catalogs of the two fields are presented in the Tables 6 and 7, which are available only in the electronic edition. In addition, catalogs for the remaining fields and their completeness limits will be posted on the Web as they become available. ${ }^{11}$

\section{DISCUSSION}

As we identify objects in each optical and near-infrared bandpass, the catalogs contain objects with a wide range of colors, ranging from the reddest objects, whether they be old or dusty, to the most actively star forming systems. The

\footnotetext{
11 These will be available at http://www.ociw.edu/lcirs/catalogs.html.
} 
TABLE 4

An Example of the ObJect Catalog of the CSDF Region

\begin{tabular}{|c|c|c|c|c|c|c|c|c|c|c|c|c|c|c|c|}
\hline $\begin{array}{l}\text { TILE } \\
(1)\end{array}$ & $\begin{array}{l}\text { ID } \\
(2)\end{array}$ & $\begin{array}{c}x \\
(3)\end{array}$ & $\begin{array}{c}y \\
(4)\end{array}$ & $\begin{array}{c}\alpha(\mathbf{J} 2000) \\
\quad(5)\end{array}$ & $\begin{array}{c}\delta(\mathbf{J} 2000) \\
(6)\end{array}$ & \multicolumn{5}{|c|}{$r_{k}$ (pixels) } & \multicolumn{5}{|c|}{$\mathrm{S} / \mathrm{G}$} \\
\hline $1 \ldots \ldots \ldots$ & 5002 & 2296 & 1498 & 033230.47 & -275014.99 & 8.65 & 10.71 & 9.38 & & & 0.21 & 0.52 & 0.57 & -1.00 & -1.00 \\
\hline $1 \ldots \ldots .$. & 5003 & 2416 & 1458 & 033230.70 & -274044.92 & 14.00 & 12.99 & 12.25 & 11.67 & 12.91 & 0.92 & 0.98 & 0.87 & 0.45 & 0.01 \\
\hline $1 \ldots \ldots \ldots$ & 5004 & 2151 & 1510 & 033231.15 & -275111.24 & 8.21 & 9.68 & $\ldots$ & $\ldots$ & $\ldots$ & 0.27 & 0.72 & -1.00 & -1.00 & -1.00 \\
\hline $1 \ldots \ldots \ldots$ & 5007 & 273 & 1488 & 033231.16 & -275123.93 & 11.44 & 11.94 & 10.68 & 10.36 & 9.63 & 0.19 & 0.83 & 0.79 & 0.14 & 0.24 \\
\hline $1 \ldots \ldots .$. & 5008 & 2400 & 1505 & 033230.09 & -275100.03 & 11.10 & 8.22 & 9.07 & $\ldots$ & $\ldots$ & 0.17 & 0.13 & 0.04 & -1.00 & -1.00 \\
\hline $1 \ldots \ldots \ldots$ & 5009 & 63 & 1522 & 033229.85 & -275106.06 & 8.96 & 10.08 & $\ldots$ & & $\ldots$ & 0.69 & 0.11 & -1.00 & -1.00 & -1.00 \\
\hline $1 \ldots \ldots \ldots$ & 5010 & 1209 & 1506 & 033230.03 & -274315.44 & 12.01 & 12.11 & 11.41 & 9.24 & $\ldots$ & 0.18 & 0.17 & 0.08 & 0.53 & -1.00 \\
\hline $1 \ldots \ldots .$. & 5011 & 484 & 1514 & 033231.21 & -275053.82 & 10.43 & 9.66 & 10.01 & $\ldots$ & $\ldots$ & 0.22 & 0.20 & 0.19 & -1.00 & -1.00 \\
\hline $1 \ldots \ldots .$. & 5012 & 16 & 1522 & 033230.51 & -274212.76 & 11.27 & 11.94 & & & $\ldots$ & 0.09 & 0.63 & -1.00 & -1.00 & -1.00 \\
\hline $1 \ldots \ldots .$. & 5017 & 2072 & 1495 & 033230.98 & -274121.14 & 10.64 & 10.99 & 16.99 & 13.42 & $\ldots$ & 0.03 & & 0.01 & 0.53 & -1.00 \\
\hline $1 \ldots \ldots \ldots$ & 5018 & 1825 & 1472 & 033231.02 & -274236.66 & 21.70 & 22.05 & 18.76 & 13.04 & 12.29 & 0.04 & & 0.03 & 0.06 & 0.01 \\
\hline $1 \ldots \ldots \ldots$ & 5019 & 799 & 1514 & 033230.75 & -274345.52 & 11.39 & 8.13 & 11.09 & $\ldots$ & $\ldots$ & 0.30 & 0.40 & 0.82 & -1.00 & -1.00 \\
\hline $1 \ldots \ldots \ldots$ & 5020 & 24 & 1493 & 033231.28 & -274923.87 & 16.40 & 19.90 & & & $\ldots$ & 0.04 & 0.01 & -1.00 & -1.00 & -1.00 \\
\hline $1 \ldots \ldots \ldots$ & 5021 & 427 & 1497 & 033 & -274317.42 & 22.85 & 24.37 & 11.38 & $\ldots$ & $\ldots$ & 0.01 & 0.01 & 0.11 & -1.00 & -1.00 \\
\hline $1 \ldots \ldots .$. & 5022 & 2265 & 1519 & 033 & -274758.48 & $\ldots$ & 50.51 & $\ldots$ & $\ldots$ & $\ldots$ & -1.00 & 0.03 & -1.00 & -1.00 & -1.00 \\
\hline $1 \ldots \ldots .$. & 5023 & 1983 & 1520 & 0332 & -274543.17 & 7.00 & 7.56 & 6.85 & $\ldots$ & $\ldots$ & 0 . & 0.04 & 0.13 & -1.00 & -1.00 \\
\hline $1 \ldots \ldots .$. & 5024 & 1726 & 1506 & 033 & -274251.17 & 10.64 & 9.41 & 7.46 & 5.51 & $\ldots$ & 0.91 & 0.95 & 0.14 & 0.69 & -1.00 \\
\hline $1 \ldots \ldots \ldots$ & 5025 & 464 & 1529 & 0332 & -273946.29 & 14.98 & 9.01 & & & $\ldots$ & 0.00 & 0.10 & -1.00 & -1.00 & -1.00 \\
\hline $1 \ldots \ldots \ldots$ & 5026 & 1831 & 1506 & 033231.08 & -274951.10 & 17.71 & 10.29 & 10.36 & 10.08 & 8.47 & 0.02 & 0.35 & 0.03 & 0.02 & 0.02 \\
\hline $1 \ldots \ldots .$. & 5027 & 782 & 1521 & 033230.53 & -274030.33 & 18.03 & 11.39 & & $\ldots$ & $\ldots$ & 0.13 & 0.04 & -1.00 & -1.00 & -1.00 \\
\hline $1 \ldots \ldots \ldots$ & 5028 & 1287 & 1529 & 033231.00 & -274030.80 & 7.80 & 12.53 & 12.34 & $\ldots$ & $\ldots$ & 0.58 & 0.64 & 0.72 & -1.00 & -1.00 \\
\hline $1 \ldots \ldots .$. & 5029 & 1929 & 1526 & 033231.46 & -275015.44 & 11.60 & 5.53 & $\ldots$ & $\ldots$ & $\ldots$ & 0.69 & 0.68 & -1.00 & -1.00 & -1.00 \\
\hline $1 \ldots \ldots \ldots$ & 5038 & 1874 & 1518 & 033231.43 & -275042.36 & 10.10 & 8.87 & 12.57 & $\ldots$ & $\ldots$ & 0.36 & 0.89 & 0.13 & -1.00 & -1.00 \\
\hline $1 \ldots \ldots \ldots$ & 5039 & 657 & 1511 & 033230.72 & -274617.22 & 9.38 & 10.72 & 7.46 & & & 0.67 & 0.27 & 0.28 & -1.00 & -1.00 \\
\hline $1 \ldots \ldots .$. & 5040 & 1211 & 1536 & 31.21 & -274310.45 & $\cdots$ & 4.58 & $\ldots$ & $\cdots$ & & -1.00 & 0.49 & -1.00 & -1.00 & -1.00 \\
\hline $1 \ldots \ldots .$. & 5041 & 1387 & 1517 & & -27485 & & 22.23 & 7.49 & 6.88 & & & 0.03 & 0.13 & 0.60 & -1.00 \\
\hline $1 \ldots \ldots \ldots$ & 5042 & 2021 & 15 & & -274157.24 & 21.10 & 10.36 & 13.65 & 19.62 & & & & 0.09 & 0.09 & -1.00 \\
\hline $1 \ldots \ldots \ldots$ & 5043 & 171 & 1536 & 033230.70 & -275024.73 & 5.44 & $\cdots$ & $\cdots$ & $\cdots$ & $\ldots$ & 0.55 & -1.00 & -1.00 & -1.00 & -1.00 \\
\hline $1 \ldots \ldots \ldots$ & 5044 & 1160 & 1503 & 033231.21 & -275034.58 & 14.02 & 11.44 & 12.59 & 12.11 & 8.32 & 0.02 & 0.02 & 0.02 & 0.01 & 0.00 \\
\hline $1 \ldots \ldots \ldots$ & 5045 & 1857 & 1529 & 033231.09 & -274528.99 & 6.34 & 4.93 & 5.25 & $\ldots$ & $\ldots$ & 0.78 & 0.59 & 0.74 & -1.00 & -1.00 \\
\hline $1 \ldots \ldots .$. & 5046 & 582 & 1531 & 033231.18 & -274507.05 & 9.85 & 7.43 & $\ldots$ & $\ldots$ & $\ldots$ & 0.55 & 0.52 & -1.00 & -1.00 & -1.00 \\
\hline $1 \ldots \ldots \ldots$ & 5047 & 2130 & 1512 & 033230.80 & -274004.30 & 9.66 & 10.50 & 11.65 & 7.90 & 6.34 & 0.89 & 0.88 & 0.70 & 0.43 & 0.33 \\
\hline $1 \ldots \ldots \ldots$ & 5048 & 237 & 1500 & 033230.79 & -274637.40 & 14.30 & 12.95 & 11.94 & 9.06 & $\ldots$ & 0.02 & 0.03 & 0.01 & 0.00 & -1.00 \\
\hline $1 \ldots \ldots \ldots$ & 5049 & 200 & 1525 & 033231.16 & -274656.21 & 9.52 & 8.99 & 5.46 & $\ldots$ & $\ldots$ & 0.30 & 0.75 & 0.44 & -1.00 & -1.00 \\
\hline $1 \ldots \ldots \ldots$ & 5050 & 1340 & 1522 & 033231.34 & -274446.32 & 10.53 & 10.54 & $\ldots$ & & $\ldots$ & 0.63 & 0.68 & -1.00 & -1.00 & -1.00 \\
\hline
\end{tabular}

NoтE.--Units of right ascension are hours, minutes, and seconds, and units of declination are degrees, arcminutes, and arcseconds.

full galaxy sample is therefore representative of galaxies of a wide range of stellar properties and redshifts, and can be used to address a variety of cosmological issues. Here we examine photometric properties of the $H$-band-selected objects and derive the number-magnitude relation for the $H$-band-selected galaxies as well as for red galaxies of different optical and near-infrared colors. We compare our measurements with various model predictions and discuss the implications.

\subsection{Distributions of Optical and Near-Infrared Colors}

We present the $I-H$ versus $H$-band color-magnitude diagram for the $\sim 14,000 \mathrm{H}$-band-selected galaxies identified in the HDF-S and CDF-S regions in Figure 9. Objects classified as stars are not included. We show the 95\% completeness limit of the $H$-band data by the short-dashed line and the $5 \sigma$ detection threshold over a $4^{\prime \prime}$ diameter aperture for the $I$-band images of the HDF-S and CDF-S regions by the 


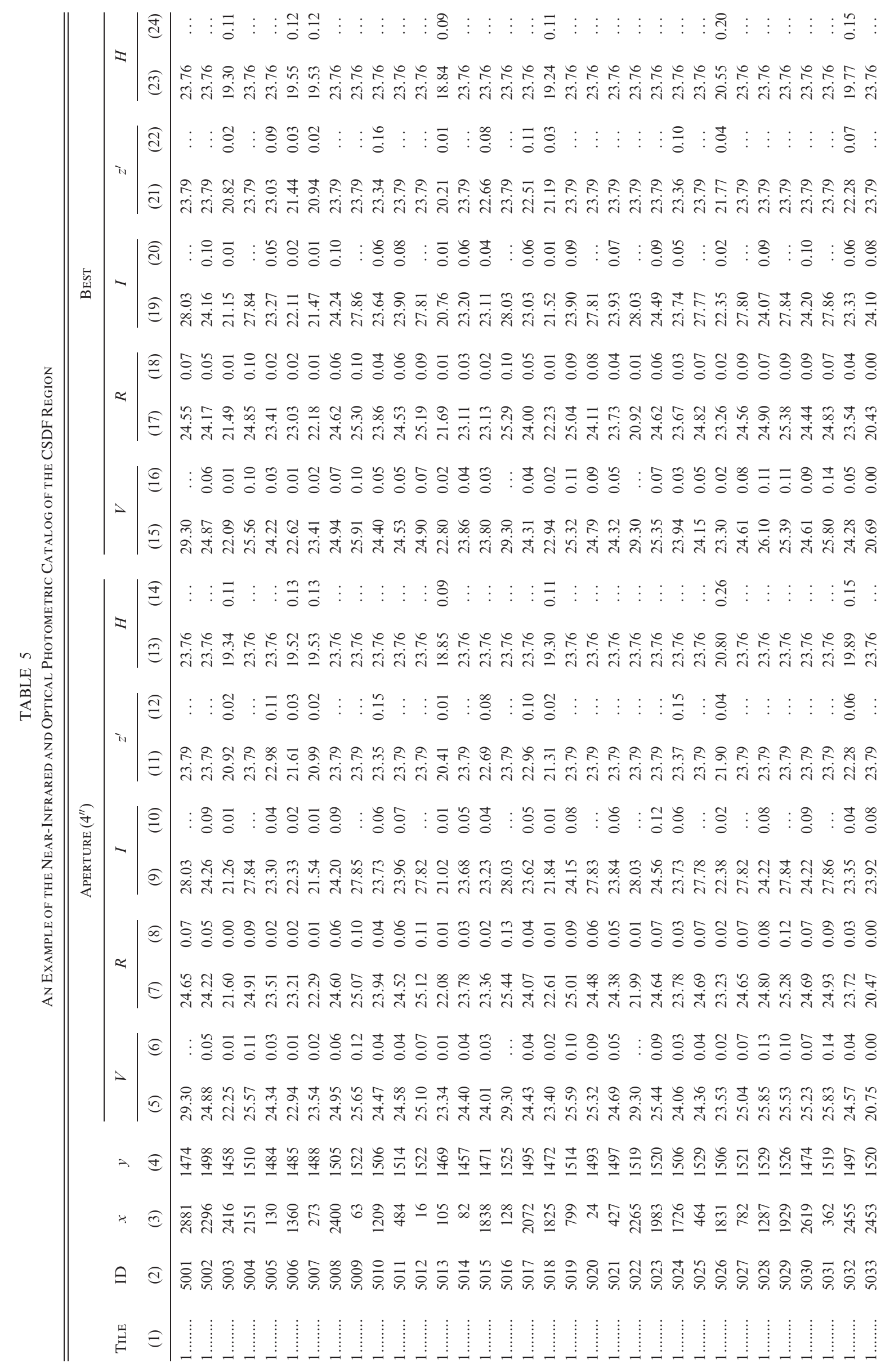




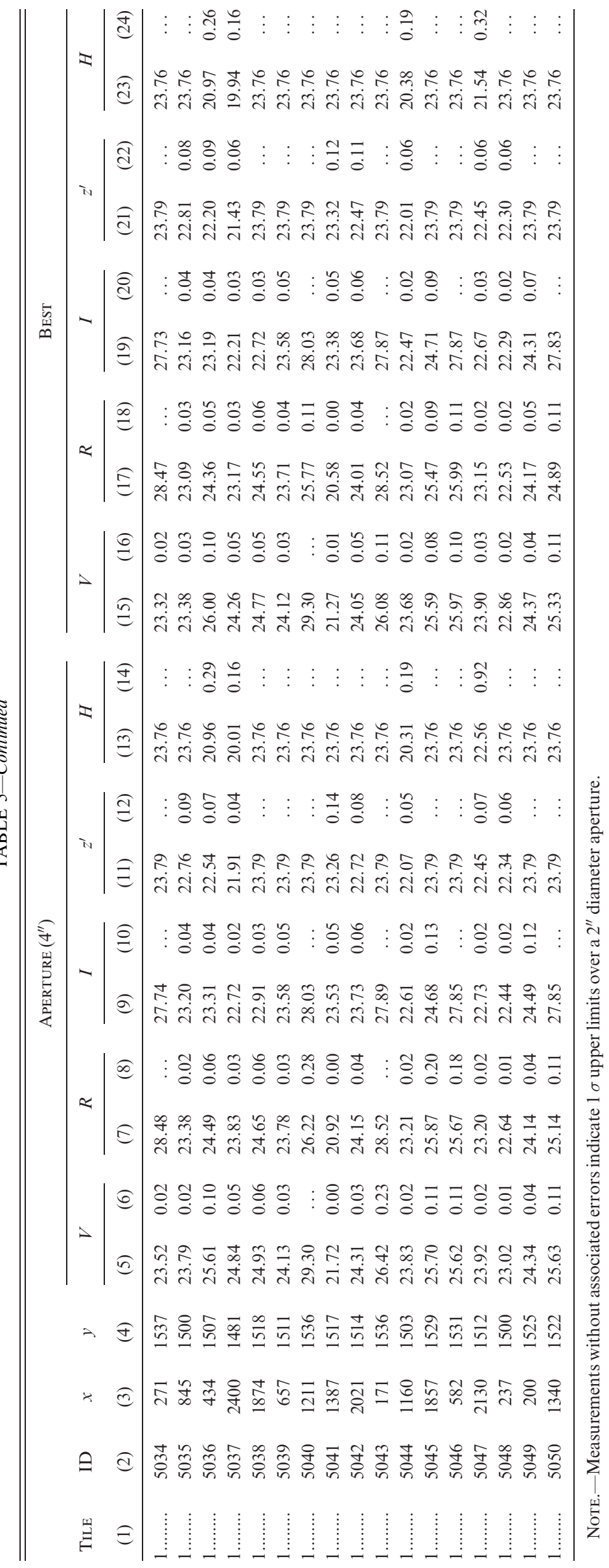


Table 6

Full Object Catalog

This table is available only on-line as a machine-readable table

dot-dashed lines. We also present the expected color-magnitude evolution of an $L_{*}$ elliptical galaxy from $z=2$ to the present time under different evolution scenarios for $h=0.7$. The curves trace the trajectories of a nonevolving elliptical galaxy spectrum (solid curve), a passively evolving galaxy formed in a single burst at $z=10$ (dash-dotted curve), and a galaxy formed at $z=30$ with an exponentially declining star formation rate $[\mathrm{SFR} \sim \exp (-t / \tau)]$ for $\tau=1$ Gyr (shortdashed curve). The long-dashed curve corresponds to a nonevolving $3 L_{*}$ early-type galaxy.

Comparisons of measurements and model predictions show that the $I-H$ colors of galaxies with $H \lesssim 18$ agree well with the expected colors at low redshifts. In addition, they show that the median color becomes progressively bluer at fainter magnitudes, accompanied by a tail of galaxies with very red colors $(I-H \gtrsim 3)$. These red galaxies constitute a negligible fraction of the total population at $H \sim 18$, but become a significant constituent by $H \sim 20$. Similar trends are seen in $B-K, R-K$ and $I-K$ color-magnitude diagrams based on smaller area surveys (Elston, Rieke, \& Rieke 1988; Cowie et al. 1994, 1996; Ellis 1997; Menanteau et al. 1999). Furthermore, the good agreements between observed colors and model predictions suggest that these red galaxies are mildly evolving early-type galaxies at $z \sim 1$ sampled over the bright end of the luminosity function $\left(\sim 1-5 L_{*}\right)$. The red sequence observed in clusters with $z \sim 0.8-1.2$ populate a similar range in the $I-H$ versus $H$ color-magnitude diagram, supporting the idea that the bulk of the red galaxies are evolved early-type galaxies at intermediate redshifts (e.g., Stanford et al. 1997, 1998; Chapman, McCarthy, \& Persson 2000).

We present the $V-I$ versus $I-H$ color-color distribution of the $H$-band-detected objects in the HDF-S and CDF-S regions in Figure 10. Stars that were classified according to the criteria described in $\S 4.5$ are indicated by crosses, and galaxies are indicated by filled circles. Open circles indicate galaxies that are not detected in $V$. The solid curves trace the trajectories of different Coleman et al. (1980) spectral templates at redshifts from $z=2$ to the present time: ellipti$\mathrm{cal} / \mathrm{S} 0$ (yellow), Sab (green), Scd (cyan), and irregular (blue). The dotted curve shows the trajectory of a passively evolving galaxy formed in a single burst at redshift $z_{f}=10$. The short- and long-dashed curves trace the colors of galaxies formed at $z_{f}=30$ with exponentially declining star formation rates for $\tau=1$ and $2 \mathrm{Gyr}$, respectively. The dash-dotted line corresponds to a galaxy formed at $z_{f}=30$ with a constant star formation rate. Filled circles along each curve indicate the corresponding redshifts, starting from $z=2$ in the lower right corner, evolving toward lower redshifts in steps of $\Delta z=0.5$, and ending at $z=0$ in the lower left corner.

Table 7

Full Photometric Catalog

\footnotetext{
This table is available only on-line as a machine-readable table
}

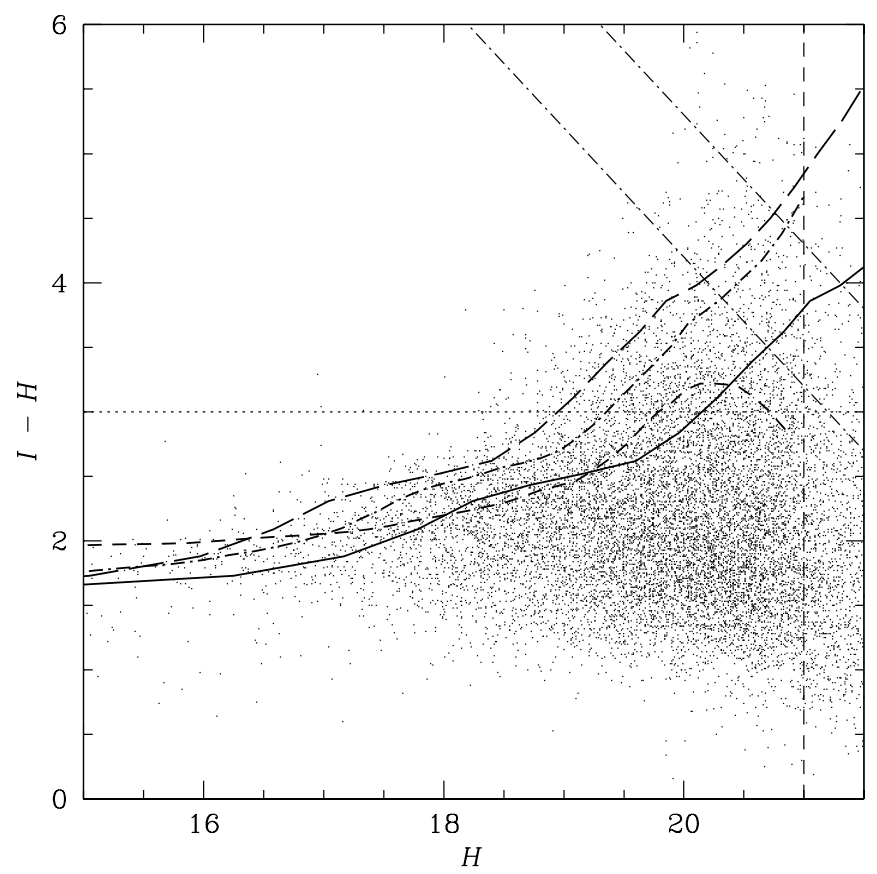

Fig. 9. $-I-H$ color vs. $H$-band magnitude diagram for the $H$-band selected galaxies in the HDF-S and CDF-S regions. Short-dashed line shows the $95 \%$ completeness limit of the $H$-band data, and the dot-dashed lines show the $5 \sigma$ detection threshold over a $4^{\prime \prime}$ diameter aperture for the $I$ band images of the HDF-S and CDF-S regions. The solid curve represents a nonevolving $L_{*}$ elliptical galaxy; the dash-dotted curve represents a passively evolving $L_{*}$ galaxy formed at $z=10$; and the short-dashed curve represents an $L_{*}$ galaxy constantly forming stars since $z=30$. The longdashed curve represents a nonevolving $3 L *$ elliptical galaxy. The dotted line indicates the $I-H=3$ color-selection threshold for red galaxies at $z \gtrsim 1$.

The general agreement between distributions of various model predictions and measurements of galaxies in our catalog, and the clear separation of the stellar sequence suggest that most stars have been accurately identified in these two survey fields. A similar separation is made in a $B-I$ versus $I-K$ color-color diagram (e.g., Gardner 1992; Huang et al. 1997). The large scatter in the $V-I$ colors spanned by the galaxies reveals a wide range of star formation histories for which our simple models provide only an idealized description. In particular, the large scatter in the $V-I$ colors for galaxies of $I-H$ colors indicative of redshifts $z \sim 1$ suggest a significant amount of ongoing star formation in these galaxies. Similar results are found in detailed studies of morphologically selected early-type galaxies at intermediate redshifts that reveal a deficit of distant ellipticals with optical and UV colors that match with expectations based on passively evolving galaxies formed at high redshifts (e.g., Menanteau et al. 1999; Menanteau, Abraham, \& Ellis $2001)$. In addition, the small number $(<15 \%)$ of galaxies with $I-H \gtrsim 3$ and $V-I \gtrsim 3$ suggests that there are few objects that can be adequately characterized as pure passively evolving systems formed at high redshifts. The trajectories of the evolving galaxy models in the color-color diagram suggests that these red galaxies span a significant range in redshift and that those of bluer $V-I$ colors largely arise at redshifts $z \gtrsim 1.5$. This is supported by their angular clustering amplitude (McCarthy et al. 2001b) and photometric redshift measurements $(H$. W. Chen et al., in preparation). 


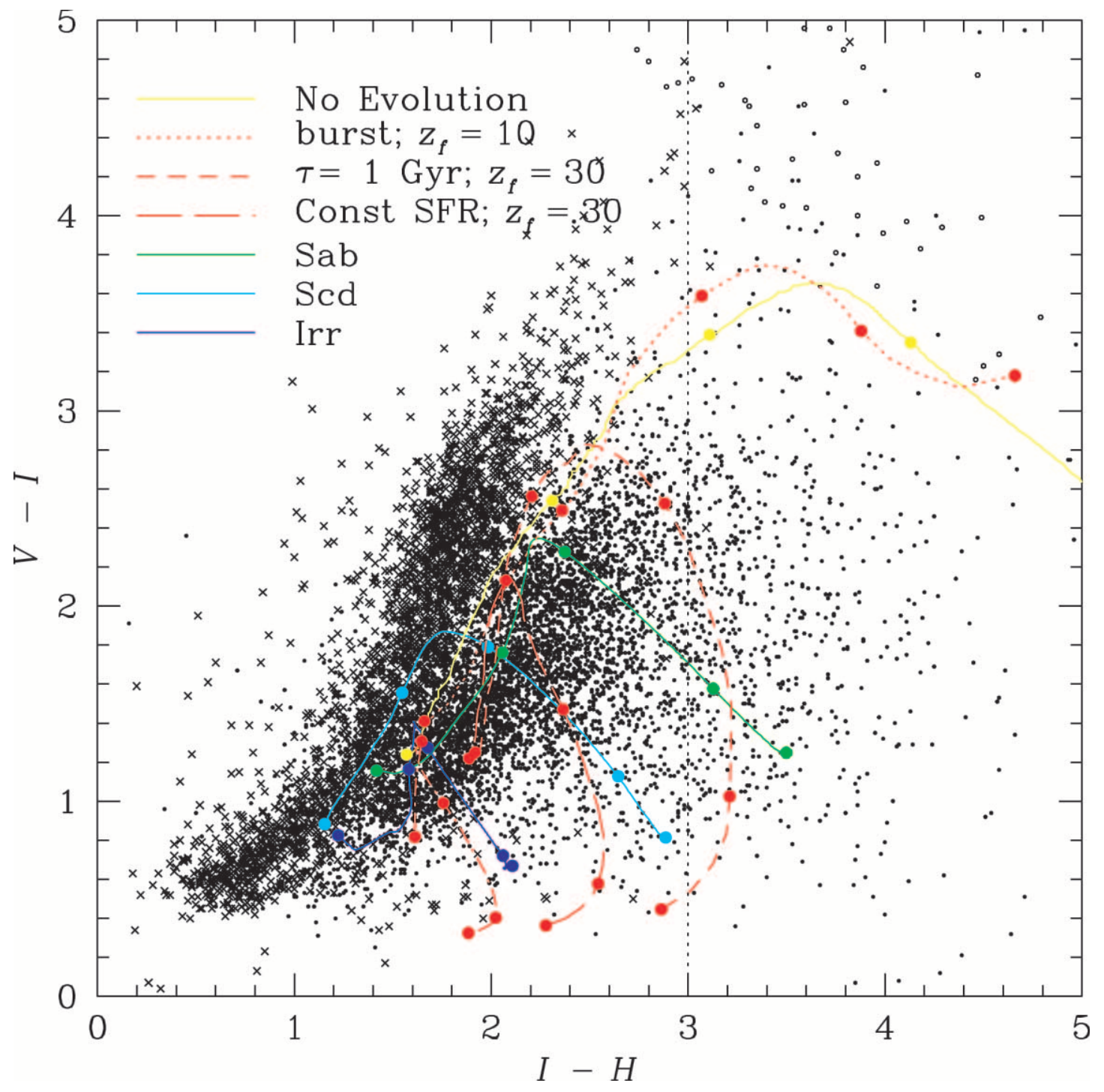

FIG. 10. $-V-I$ vs. $I-H$ color-color diagram for objects identified as stars (crosses) and galaxies (filled circles) in the HDF-S and CDF-S regions. Open circles indicate galaxies that are not detected in $V$. Solid curves indicate the mean colors of galaxy of different types as a function of redshift at redshifts between $z=0$ and 2, from elliptical or S0 (yellow), Sab (green), Scd (cyan), to irregular (blue) galaxies. The dotted curve indicates the predicted colors of a passively evolving galaxy formed in a single burst at redshift $z_{f}=10$. The short- and long-dashed curves indicate the predicted colors of galaxies formed at $z_{f}=30$ with an exponentially declining star formation rate $\exp (-t / \tau)$ for $\tau=1$ and $2 \mathrm{Gyr}$, respectively. The dash-dotted line indicates the predicted colors of a galaxy formed at $z_{f}=30$ with a constant star formation rate at all redshifts. Filled circles along each curve indicate the corresponding redshifts, starting from $z=2$ on the right and evolving toward lower redshifts in steps of $\Delta z=0.5$. The dotted line indicates the $I-H=3$ color-selection threshold for red galaxies at $z \gtrsim 1$.

\subsection{The H-Band Number Counts}

Galaxy number counts provide a diagnostic for distinguishing between different evolutionary scenarios for galaxies of different colors (e.g., Metcalfe et al. 1996). Comparisons of number-count measurements from different surveys also allow us to examine the accuracy of our photometric calibration. Figure 11 shows the $H$-band differential galaxy counts $N(m)$ derived from $\sim 14,000$ galaxies selected in the $H$-band survey toward the HDF-S and CDF$\mathrm{S}$ regions (filled circles). We applied incompleteness corrections down to the $95 \%$ completeness level using the incompleteness curves determined for compact and extended sources in each tile. According to the procedures described in $\S 4.3$, we adopted the stellarity index determined in SExtractor for each object and corrected for the incompleteness at faint magnitudes using the compact source incomplete- ness curve for objects of stellarity index greater than 0.5 and using the extended source incompleteness curve otherwise. The vertical bars indicate the uncertainties in our measurements estimated using a variation of the "bootstrap" resampling technique. The bootstrap resampling technique explicitly accounts for the sampling error and for photometric uncertainties. We first resampled objects from the parent catalog (allowing for the possibility of duplication) and added random noise (within the photometric uncertainties) to the photometry. Then we estimated the $H$-band differential number counts for the resampled, perturbed catalog. Finally, we repeated the procedures 1000 times and determined the $1 \sigma$ uncertainties based on the 16th and 84th percentiles of the distributions in each magnitude bin.

For comparison, we have also included in Figure 11 previous $H$-band measurements reported by various groups. Our measurements span 6 mag in $H$ and nicely bridge the 


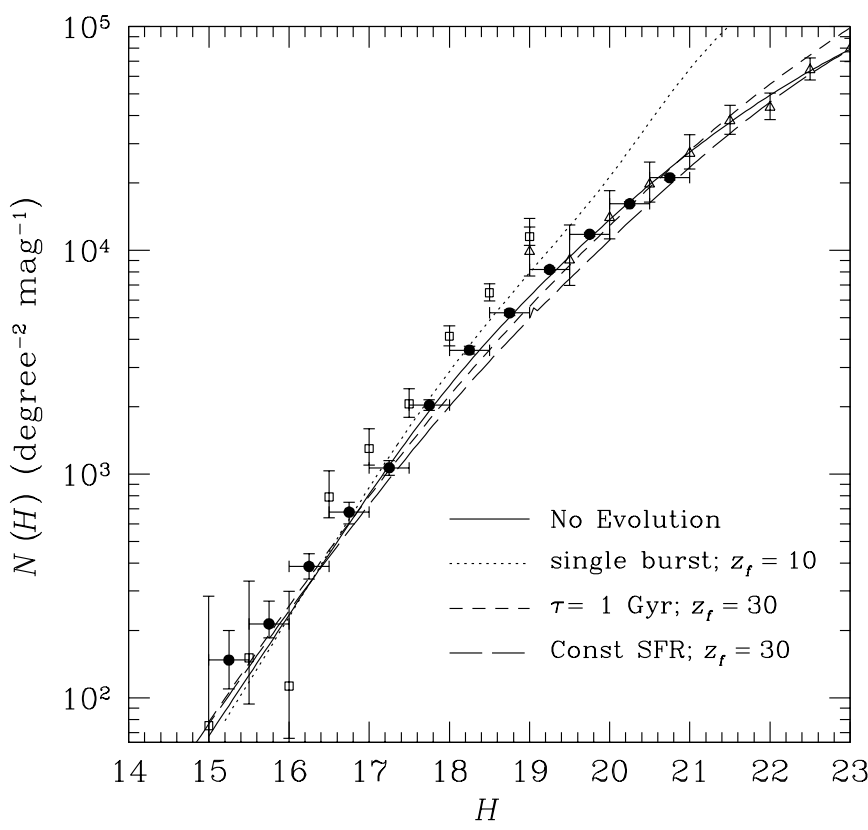

FIG. 11.-Differential number counts for the $H$-band-selected galaxies in the HDF-S and CDF-S regions. The open symbols show previous measurements presented by Yan et al. (1998; triangles) and Martini (2001b; squares). The curves are predictions based on various star formation scenarios discussed in $\S 6.2$.

gap between the existing measurements at the bright (open squares; from Martini 2001b) ${ }^{12}$ and faint ends (Yan et al. 1998). The excellent agreements in both the slopes and the normalization demonstrate not only the accuracy of our $H$-band photometry, but also the accuracy in our star and galaxy separation. The best-fitting power-law slopes, $d \log N(m) / d m$, were found to be $0.45 \pm 0.01 \mathrm{mag}^{-2}$ at $H \lesssim 19$ and $0.27 \pm 0.01 \mathrm{mag}^{-2}$ at $H \gtrsim 19$, with a mean surface density of $\approx 7200 \mathrm{deg}^{-2} \mathrm{mag}^{-1}$ at $H=19 .{ }^{13}$ The slope of the faint-end $H$-band counts is in line with that of the faint-end $K$-band counts (Cowie et al. 1994; Djorgovski et al. 1995; Bershady, Lowenthal, \& Koo 1998; Gardner et al. 1996; Metcalfe et al. 1996; McCracken et al. 2000). The slope of the bright-end $H$-band counts $(H \lesssim 19)$ is slightly shallower than that of the bright-end $K$-band counts reported by Huang et al. (1997) and Metcalfe et al. (1996), although we note that our counts are not well determined at $H<16$.

To compare with simple model predictions, we considered a no-evolution model based on an early-type galaxy template (Fig. 11, solid curve), a passive evolution scenario for galaxies formed in a single burst at $z_{f}=10$ (dotted curve), an exponentially declining star formation for galaxies formed at $z_{f}=30$ (short-dashed curve), and a constant

\footnotetext{
12 Part of the apparent excess in the number count measurements by Martini (2001b) is due to an additional aperture correction that the author applied in the galaxy photometry to account for missing light in the wings of galaxy profiles, which was found to be between 0.1 and $0.2 \mathrm{mag}$.

${ }^{13}$ We note that the uncertainties in the number count measurements due to random errors are small, given the large sample from our survey, but systematic errors are likely to dominate and are more difficult to measure. In particular, number-count measurements at the faintest magnitude bins are more likely to be overestimated because of photometric uncertainties in the even fainter and more abundant populations. As a result, the faint-end slope is likely to be overestimated.
}

star formation for galaxies formed at $z_{f}=30$ (long-dashed curve). We adopted the $K$-band luminosity function derived by Gardner et al. (1997) and applied an $H-K \approx 0.22$ color correction (Mobasher, Ellis, \& Sharples 1986) to obtain an estimate for $M_{H_{*}}$. This is appropriate for galaxies of different type because the $k$-correction is nearly independent of spectral type at near-infrared wavelengths. We find that all but the passive evolution scenario (Fig. 11, dotted curve) agree well with the measurements.

\subsection{Surface Density of Red Galaxies}

In this section, we present surface density estimates of moderately red galaxies of $I-H \gtrsim 3$ and compare the measurements with those of extremely red objects (EROs) identified in the survey. EROs are typically selected based on their extreme optical and near-infrared colors $R-K \gtrsim 6$ (e.g., Elston et al. 1988; McCarthy, Persson, \& West 1992; $\mathrm{Hu} \&$ Ridgway 1994). Here we selected EROs using $R-H \gtrsim 5$, which according to the simulations presented in Figure 1 is equivalent to $R-K \gtrsim 6$ for high-redshift evolved populations.

Figure 12 shows the $H$-band differential number counts for galaxies of $I-H \geq 3$ (filled circles) and for galaxies of $R-H \gtrsim 5$ (squares) identified in the HDF-S and CDF-S regions. Incompleteness corrections were applied at faint magnitudes, and the uncertainties indicated by the vertical bars were estimated following the same procedures described in $\S 6.2$. The slope of the red counts is quite steep, $d \log N(m) / d m=0.84 \pm 0.06 \mathrm{mag}^{-2}$ for galaxies of $I-H \gtrsim 3$ and $0.89 \pm 0.24 \mathrm{mag}^{-2}$ for galaxies of $R-H \gtrsim 5$ at $H \lesssim 20$. The counts appear to have shallower faint-end slopes for both of the red subsamples-between 0.17 and $0.32 \mathrm{mag}^{-2}$ - at $H \gtrsim 20$. While both subsamples show con-

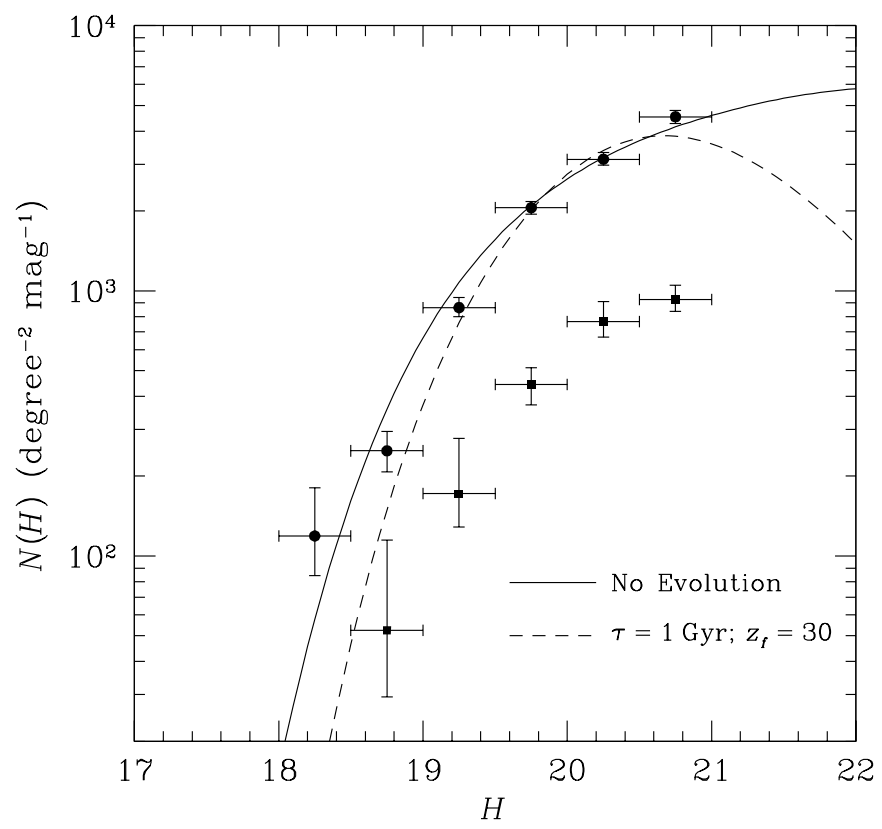

FIG. 12.-Differential number counts for the $H$-band-selected red galaxies in the HDF-S and CDF-S regions. Filled circles show galaxies of $I-H \gtrsim 3$, and squares show galaxies of $R-H \gtrsim 5$. As shown in Fig. 11, the solid curve indicates the prediction based on a nonevolving scenario, and the short-dashed curve indicates an exponentially declining star formation scenario for galaxies formed at $z_{f}=30$. These models are described in $\S 6.3$. 
sistent slopes at the bright end, they have a more steeply rising $N(m)$ with the $H$ magnitude than the total $H$-banddetected population. The steeper bright-end slope of the red galaxies indicates that most of the foreground $(z \lesssim 1)$ galaxies have been effectively excluded from the red samples and that the shape of the number counts reflects the shape of the bright-end luminosity function of the red population. In addition, the consistent slopes between the two red subsamples suggest that the underlying luminosity function is very similar.

For comparison, we also calculated the predicted differential number counts for red galaxies with $I-H \geqslant 3$ based on the no-evolution scenario and the exponentially declining star formation scenario with $\tau=1$ Gyr that best fit the observed total $H$-band number counts, as discussed in $\S 6.2$. To model the intrinsic luminosity function of evolved galaxies, we adopted the population ratio of elliptical galaxies in the local universe (e.g., Binggeli, Sandage, \& Tammann 1988). We first scaled the $K$-band luminosity function obtained by Gardner et al. (1997) for the total galaxy population by $M_{*}($ red $)=M_{*}($ tot $)-0.2$ and $\phi_{*}($ red $)=$ $0.2 \phi_{*}$ (tot) and then assumed a faint-end slope of $\alpha=1$. The curves indicate the results of the calculations and are the same as those in Figure 11. It appears that both scenarios fit the observations fairly well, but that the exponentially declining star formation model predicts a sharp turnover in the differential number counts at $H \gtrsim 21$. The turnover is due to the fact that at these faint magnitudes the evolved population is dominated by those at redshifts $z \gtrsim 2$, where they would have bluer $I-H$ colors under the exponentially declining star formation scenario and would therefore be excluded based on the $I-H \gtrsim 3$ selection criterion (see Fig. 9). Because of large uncertainties in the optical and $H$-band photometry for galaxies at these faint magnitudes, we cannot rule out either of the scenarios. The moderately good agreements between observations and model predictions, together with the large scatter in the optical $V-I$ color discussed in $\S 6.1$, however, suggest that there is still some amount of star formation going on in the red population at redshifts $z>1$.

In Figure 13 we plot the cumulative surface density measurements of galaxies with $I-H \geq 3$ (filled circles) and of $R-H \gtrsim 5$ (squares) as a function of $H$-band magnitude, in comparison with those of the total $H$-band-selected galaxies and with previous measurements of EROs presented by Daddi et al. (2000; open squares), Yan et al. (2000; open triangles), and Thompson et al. (1999; cross). Our surface density measurements of EROs span 2 mag in $H$ and are consistent with those from previous surveys. In addition, we find that red galaxies with $I-H \gtrsim 3$ constitute $\approx 20 \%$ of the $H$-band-detected galaxies at $H \lesssim 21$, but only $\approx 2 \%$ at $H \lesssim 19$. Specifically, we expect to observe on average $\sim 40$ galaxies of $I-H \gtrsim 3$ and $\sim 8$ galaxies of $R-H \gtrsim 5$ among $\sim 270 \mathrm{H}$-band-detected galaxies at $H \lesssim 21$, but only $\sim 1$ galaxy of $I-H \gtrsim 3$ and less than 1 of $R-H \gtrsim 5$ among $\sim 46$ $H$-band-detected galaxies at $H \lesssim 19$ in a random 25 arcmin $^{2}$ region.

On the other hand, because EROs and these moderately red galaxies are found to exhibit strong angular clustering (Daddi et al. 2000; McCarthy et al. 2001b; Firth et al. 2002, in preparation), a large dispersion in the surface density measurements is expected. To examine the accuracy of the surface density measurements presented in Figure 13, we estimated the field-to-field variation by measuring the sur-

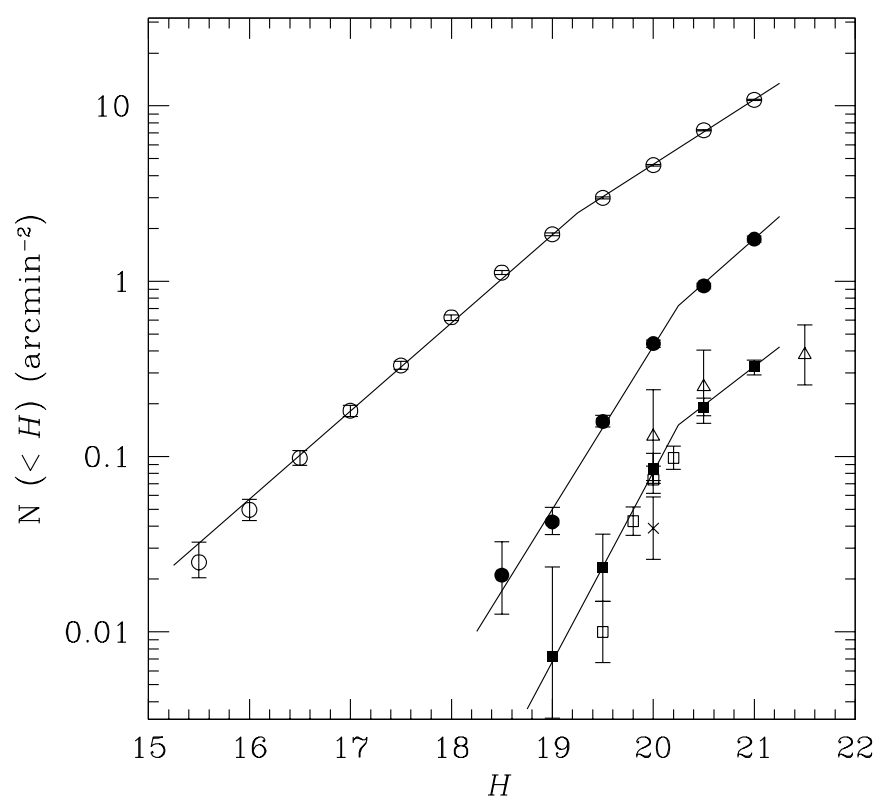

FIG. 13.-Cumulative surface densities of the total $H$-band-detected galaxies (open circles) and galaxies of $I-H \gtrsim 3$ (filled circles) and of $R-H \gtrsim 5$ (squares) as a function of $H$-band magnitude. The open squares are measurements presented by Daddi et al. (2000); the open triangles are from Yan et al. (2000); and the cross is from Thompson et al. (1999). The solid lines show the best-fit power laws to the cumulative counts.

face density of galaxies of $I-H \gtrsim 3$ in a randomly selected $5 \times 5 \operatorname{arcmin}^{2}$ region in each tile and compared the measurements between different tiles. We found that the total number of red galaxies of $H \lesssim 20.5$ varies from $\approx 15$ to $\approx 40$, with a mean surface density of $\approx 1 \operatorname{arcmin}^{-2}$ and an rms dispersion of $\approx 0.4 \mathrm{arcmin}^{-2}$. The mean surface density estimated based on the randomly selected smaller areas is consistent with the measurements presented in Figure 12 and the red counts over $0.62 \mathrm{deg}^{2}$ presented in McCarthy et al. (2001a, 2001b), indicating that our surface density measurements are not significantly affected by the cosmic variance due to clustering. In addition, following Roche et al. (1999), we estimate an angular clustering amplitude of these red galaxies to be $A \approx 4$ at $1^{\prime \prime}$ (corresponding to a correlation angle of $\theta_{0} \approx 5^{\prime \prime}$ ), which indeed agrees well with the results of an angular clustering analysis presented in McCarthy et al. (2001b). We therefore conclude that a deep, wide-field survey is necessary not only to identify a large number of red galaxies, but also to obtain accurate estimates of their statistical properties.

\section{SUMMARY}

We present initial results from the Las Campanas Infrared Survey, which is a wide-field near-infrared and optical imaging survey designed to reliably identify a statistically significant and representative sample of evolved galaxies at redshifts $z>1$ and to secure a uniform sample of galaxies of all types at intermediate redshifts. We present sample nearinfrared and optical photometric catalogs for objects identified in two of the six survey fields, surrounding the Hubble Deep Field-South and Chandra Deep Field-South regions. We have identified $\sim 14,000 H$-band-detected galaxies over 
$1408 \mathrm{arcmin}^{2}$ of sky, $\sim 2000$ of which are likely to be evolved galaxies at $z \gtrsim 1$ with $I-H \gtrsim 3$. Furthermore, we find that:

1. Red galaxies with $I-H \gtrsim 3$ are a significant constituent of galaxies with $H \gtrsim 20$ and are consistent with mildly evolving early-type galaxies at $z \sim 1$. However, there exists a large scatter in the $V-I$ colors spanned by the red galaxies, indicating a significant amount of ongoing star formation in these galaxies.

2. The differential number counts $N(m)$ for the $H$-banddetected objects has a slope of $d \log N(m) / d m=0.45 \pm 0.01$ $\mathrm{mag}^{-2}$ at $H \lesssim 19$ and $0.27 \pm 0.01 \mathrm{mag}^{-2}$ at $H \gtrsim 19$, with a mean surface density $\approx 7200 \mathrm{deg}^{-2} \mathrm{mag}^{-1}$ at $H=19$.

3. The differential number counts for red objects $(I-H \gtrsim 3)$ detected in the $H$-band has a very steep slope, $d \log N(m ; I-H \gtrsim 3) / d m=0.84 \pm 0.06 \mathrm{mag}^{-2}$ at $H \lesssim 20$ and $0.32 \pm 0.07 \mathrm{mag}^{-2}$ at $H \gtrsim 20$, with a mean surface density $\approx 3000 \mathrm{deg}^{-2} \mathrm{mag}^{-1}$ at $H=20$.

4. Red galaxies $(I-H \gtrsim 3)$ constitute $\approx 20 \%$ of the $H$ band-detected galaxies at $H \lesssim 21$, but only $\approx 2 \%$ at $H \lesssim 19$. This suggests that foreground galaxies $(z \lesssim 1)$ can be effectively excluded from the total $H$-band population by applying the $I-H$ color-selection criterion and that the steep bright-end slope of red galaxy number counts reflects the shape of the bright-end luminosity function of the red population.

5. Red galaxies are strongly clustered, producing strong field-to-field variation in their surface density. We estimate a mean surface density of $\approx 1 \mathrm{arcmin}^{-2}$, with an rms dispersion of $\approx 0.4 \operatorname{arcmin}^{-2}$ over $5 \times 5 \operatorname{arcmin}^{2}$ sky regions for galaxies with $H \lesssim 20.5$ and $I-H \gtrsim 3$, implying an angular clustering amplitude of $A \approx 4$ at $1^{\prime \prime}$ and a correlation angle of $\theta_{0} \approx 5^{\prime \prime}$.

We are grateful to Xiaohui Fan, Sandy Leggett, and Ben Oppenheimer for providing various digitized stellar templates of M dwarfs, L dwarfs, and T dwarfs, and to Ken Lanzetta for providing galaxy templates for the photometric redshift analysis. We appreciate the expert assistance from the staffs of the Cerro Tololo Inter-American Observatory and Las Campanas Observatory. This research was supported by the National Science Foundation under grant AST 99-00806. The CIRSI camera was made possible by the generous support of the Raymond and Beverly Sackler Foundation.

\section{REFERENCES}

Barger, A. J., Cowie, L. L., Trentham, N., Fulton, E., Hu, E. M., Songaila, A., \& Hall, D. 1999, AJ, 117, 102

Beckett, M. G., Mackay, C. D., McMahon, R. G., Parry, I. R., Ellis, R. S., Chan, S. J., \& Hoenig, M. 1998, Proc. SPIE, 3354, 14

Benítez, N., Broadhurst, T. J., Bouwens, R. J., Silk, J., \& Rosati, P. 1999, ApJ, 515, L65

Bershady, M., Lowenthal, J., \& Koo, D. C. 1998, ApJ, 505, 50

Bertin, E., \& Arnouts, S. 1996, A\&AS, 117, 393

Binggeli, B., Sandage, A., \& Tammann, G. A. 1988, ARA\&A, 26, 509

Blain, A. W., Kneib, J.-P., Ivison, R. J., \& Smail, I. 1999, ApJ, 512, L87

Broadhurst, T. J., \& Bouwens, R. J. 2000, ApJ, 530, L53

Bruzual, A. G., \& Charlot, S. 1993, ApJ, 405, 538

Chapman, S. C., McCarthy, P. J., \& Persson, S. E. 2000, AJ, 120, 1612

Coleman, G. D., Wu, C. C., \& Weedman, D. W. 1980, ApJS, 43, 393

Connolly, A. J., Szalay, A. S., Dickinson, M., Subbarao, M. U., \& Brunner, R. J. 1997, ApJ, 486, L11

Cowie, L. L., Gardner, J. P., Hu, E. M., Songaila, A., Hodapo, K.-W., \& Wainscoat, R. J. 1994, ApJ, 434, 114

Cowie, L. L., Songaila, A., Hu, E., \& Cohen, J. G. 1996, AJ, 112, 839

da Costa, L. N. 2001, in From Extrasolar Planets to Cosmology: VLT

Opening Symposium, ed. J. Bergeron \& A. Renzini (New York: Springer), 192

da Costa, L., et al. 2001, A\&A, submitted (astro-ph/9812190)

Daddi, E., Cimmatti, A., Pozzetti, L., Hoekstra, H., Röttgering, H. J. A., Renzini, A., Zamorani, G., \& Mannucci, F. 2000, A\&A, 361, 535

Dalcanton, J. J. 1998, ApJ, 495, 251

De Propris, R., Eisenhardt, P. R., Stanford, S. A., \& Dickinson, M. 1998, ApJ, 503, L45

Djorgovski, S., et al. 1995, ApJ, 438, L13

Eggen, O. J., Lynden-Bell, D., \& Sandage, A. R. 1962, ApJ, 136, 748

Ellis, R. S. 1997, ARA\&A, 35, 389

Elston, R. 1998, Proc. SPIE, 3354, 404

Elston, R., Rieke, G. H., \& Rieke, M. J. 1988, ApJ, 331, L77

Fan, X., et al. 2000, AJ, 119, 928

Fernández-Soto, A., Lanzetta, K. M., Chen, H.-W., Pascarelle, S. M., \& Yahata, N. 2001, ApJS, 135, 41

Fernández-Soto, A., Lanzetta, K. M., \& Yahil, A. 1999, ApJ, 513, 34

Franceschini, A., Silva, L., Fasano, G., Granato, L., Bressan, A., Arnouts, S., \& Danese, L. 1998, ApJ, 506, 600

Fukugita, M., Shimasaku, K., \& Ichikawa, T. 1995, PASP, 107, 945

Gardner, J. P. 1992, Ph.D. thesis, Univ. Hawaii

Gardner, J. P., Sharples, R. M., Carrasco, B. E., \& Frenk, C. S. 1996, MNRAS, 282, L1

Gardner, J. P., Sharples, R. M., Frenk, C. S., \& Carrasco, B. E. 1997, ApJ, 480, L99

Graham, J. R., \& Dey, A. 1996, ApJ, 471, 720

Hu, E. M., \& Ridgway, S. E. 1994, AJ, 107, 1303

Huang, J.-S., Cowie, L. L., Gardner, J. P., Hu, E. M., Songaila, A., \& Wainscoat, R. J. 1997, ApJ, 476, 12

Kauffmann, G., \& Charlot, S. 1998, MNRAS, 297, L23

Kauffmann, G., Charlot, S., \& White, S. D. M. 1996, MNRAS, 283, $117 \mathrm{~L}$
Kron, R. G. 1980, ApJS, 43, 305

Landolt, A. U. 1992, AJ, 104, 340

Lanzetta, K. M., Fernández-Soto, A., \& Yahil, A. 1998, in The Hubble Deep Field: Proc. Space Telescope Science Institute 1997 May Symp., ed. M. Livio, S. M. Fall, \& P. Madau (Cambridge: Cambridge Univ. Press), 143

Leggett, S. K., Allard, F., Dahn, C., Hauschildt, P. H., Kerr, T. H., \& Rayner, J. 2000, ApJ, 535, 965

Martini, P. 2001a, AJ, 121, 2301 . $2001 \mathrm{~b}$, AJ, 121,598

Marzke, R. O., et al. 1999, in ASP Conf. Ser. 191, Photometric Redshifts and High Redshift Galaxies, ed. R. Weymann et al. (San Francisco: ASP), 148

McCarthy, P. J., Persson, S. E., \& West, S. C. 1992, ApJ, 386, 52

McCarthy, P. J., et al. 2001a, in Deep Fields: Proc. ESO Workshop, ed. S. Cristiani, A. Renzini, \& R. E. Williams (Berlin: Springer), in press 2001b, ApJ, 560, L131

McCracken, H. J., Metcalfe, N., Shanks, T., Campos, A., Gardner, J. P., \& Fong, R. 2000, MNRAS, 311, 707

Menanteau, F., Abraham, R. G., \& Ellis, R. S. 2001, MNRAS, 322, 1

Menanteau, F., Ellis, R. S., Abraham, R. G., Barger, A. J., \& Cowie, L. L. 1999, MNRÄS, 309, 208

Metcalfe, N., Shanks, T., Campos, A., Fong, R., \& Gardner, J. P. 1996, Nature, 383, 236

Mobasher, B., Ellis, R. S., \& Sharples, R. M. 1986, MNRAS, 223, 11

Moffat, A. F. J. 1969, A\&A, 3, 455

Moorwood, A., Cuby, J. G., \& Lidman, C. 1998, Messenger, 91, 9

Oppenheimer, B. R., Kulkarni, S. R., Matthews, K., \& van Kerkwijk, M. H. 1998, ApJ, 502, 932

Persson, S. E., Murphy, C. D., Gunnels, S., Birk, C., Bagish, A., \& Koch, E. 2001, AJ, submitted

Persson, S. E., Murphy, D. C., Krzeminski, W., Roth, M., \& Rieke, M. J. 1998, AJ, 116, 2475

Pickles, A. J. 1998, PASP, 110, 863

Roche, N., Eales, S. A., Hippelein, H., \& Willott, C. J. 1999, MNRAS, 306, 538

Ryan, S. G. 1989, AJ, 98, 1693

Sabbey, C. N., McMahon, R. G., Lewis, J. R., \& Irwin, M. J. 2001, in ASP Conf. Ser. 238, Astronomical Data Analysis and Software Systems X, ed. F. R. Harnden, Jr., F. A. Primini, \& H. E. Payne (San Francisco: ASP), 317

Sawicki, M. J., Lin, H., \& Yee, H. K. C. 1997, AJ, 113, 1

Schade, D., Carlberg, R. G., Yee, H. K. C., \& López-Cruz, O. 1996, ApJ, 465, L103

Schade, D., et al. 1999, ApJ, 525, 31

Smail, I., Ivison, R. J., \& Blain, A. W. 1997, ApJ, 490, L5

Stanford, S. A., Eisenhardt, P. R., \& Dickinson, M. 1998, ApJ, 492, 461

Stanford, S. A., Elston, R., Eisenhardt, P. R., Spinrad, H., Stern, D., \& Dey, A. 1997, AJ, 114, 2232

Steidel, C. C., Adelberger, K. L., Giavalisco, M., Dickinson, M., \& Pettini, M. 1999, ApJ, 519, 1

Stoughton, C., et al. 2002, AJ, 123, 485 
Teplitz, H., Hill, R. S., Malumuth, E. M., Collins, N. R., Gardner, J. P., Palunas, P., \& Woodgate, B. E. 2001, ApJ, 548, 127

Thompson, D., et al. 1999, ApJ, 523, 100

Totani, T., \& Yoshii, J. 1998, ApJ, 501, L177

White, S. D. M., \& Rees, M. J. 1978, MNRAS, 183, 341
Yan, L., McCarthy, P. J., Storrie-Lombardi, L. J., \& Weymann, R. J. 1998, ApJ, 503, L19

Yan, L., McCarthy, P. J., Weymann, R. J., Malkan, M. A., Teplitz, H. I., Storrie-Lombardi, L. J., Smith, M., \& Dressler, A. 2000, AJ, 120, 575

Zepf, S. E. 1997, Nature, 390, 377 